\title{
Discontinuous Galerkin time discretization in elastoplasticity: motivation, numerical algorithms, and applications
}

\author{
Jochen Alberty ${ }^{\text {a }}$, Carsten Carstensen ${ }^{b, *}$ \\ a Mathematischen Seminar der Lehrstuhl für Wissenschaftliches Rechnen, Christian-Albrechts-Universität zu Kiel, \\ Ludewig-Meyn-Str. 4, D-24098 Kiel, Germany \\ ${ }^{\mathrm{b}}$ Institute for Applied Mathematics and Numerical Analysis, Vienna University of Technology, \\ Wiedner Hauptstrasse 8-10, A-1040 Vienna, Austria
}

Received 27 November 2001

\begin{abstract}
Discontinuous Galerkin discretizations promise to become a very flexible tool in $h p$-adaptive space-time discretizations. This is very attractive for moving interphase problems such as the free boundary between the elastic and plastic phase in elastoplastic time evolution. The mathematical model of which involves variational inequalities and so the distributional time derivative is not obviously generalized to discontinuous test functions. This paper motivates and introduces a discontinuous Galerkin $(\mathrm{dG})$ time discretization. Solution algorithms and examples are established which support feasibility and accuracy of the proposed schemes $\mathrm{dG}(0)$ and $\mathrm{dG}(1)$. The methods are compared with a backward Euler and Crank-Nicholson scheme.

(C) 2002 Published by Elsevier Science B.V.
\end{abstract}

Keywords: Elastoplasticity; Variational inequality; Plasticity with hardening; Primal problem; Dual problem; Discontinuous Galerkin method; Time discretization

\section{Introduction}

The numerical simulation of elastoplastic evolution problems is even today a challenge in the core of computational mechanics. Backward Euler (bE), Crank-Nicholson (CN), or other (generalized) mid-point rules yield time discretizations followed by a finite element space-discretization in each time step [12,20]. The second-order schemes appear less stable and not always superior to the robust implicit Euler method. Hence, higher order methods are not frequently employed in practise. Moreover, it seems false to believe that a mid-point rule generates results which are always superior to, or more accurate than, those of a $b E$

\footnotetext{
${ }^{*}$ Corresponding author. Tel.: +43(1)58801-11525; fax: +43(1)58801-11599.

E-mail addresses: jochen@fuh-alberty.de (J. Alberty), carsten.carstensen@tuwien.ac.at (C. Carstensen).
} 
scheme. The lack of higher order regularity [17,18] is only partly responsible for this. We refer, e.g., to [2] for a counter example where one time step of the first-order scheme is exact and the $\mathrm{CN}$ scheme is not. Our interpretation is that the implicit Euler scheme has additional exactness properties which make it favorable in some examples.

A hierarchy of time discretizations is desirable, e.g., to access the time discretization and so steer the time-step size within time adaptive algorithms. In this paper we propose discontinuous Galerkin schemes (abbreviated $\mathrm{dG}$ ) of order $0,1,2,3, \ldots$ and compare them with difference schemes for time discretization. The Fig. 1 displays a stress component $\sigma_{11}$ at a point in the time interval $0 \leqslant t \leqslant 1$ computed with the implicit or bE scheme, the $\mathrm{CN}$ scheme, and the discontinuous Galerkin schemes $\mathrm{dG}(0)$ and $\mathrm{dG}(1)$ for an example discussed in Section 6. Therein, one observes oscillations for $\mathrm{CN}$ after five time steps when the material behavior becomes inelastic. The approximations from $\mathrm{dG}(0)$ are drawn as horizontal line segments, those of $\mathrm{dG}(1)$ as piecewise affine segment. The curve is non-monotone and one might speculate whether bE gives the best result. Although the exact solution is unknown to us, $b E$ appears more accurate then $\mathrm{CN}$, while $\mathrm{dG}(1)$ seems superior to $\mathrm{dG}(0)$. We conjecture that the jumps of $\mathrm{dG}(1)$ are a proper indicator for smaller time steps. Discontinuous Galerkin methods hence are very desirable for adaptive multilevel error assessments. The main difficulty in their design is the correct interpretation of a time derivative of a non-smooth function or even a discontinuous function. This is much more involved than for time evolution equations $[19,21]$.

The aim of this paper is to design $\mathrm{dG}(k)$ time discretizations for elastoplastic evolution problems and prove that the methods $\mathrm{dG}(0)$ and $\mathrm{dG}(1)$ are feasible. We derive numerical algorithms and establish some

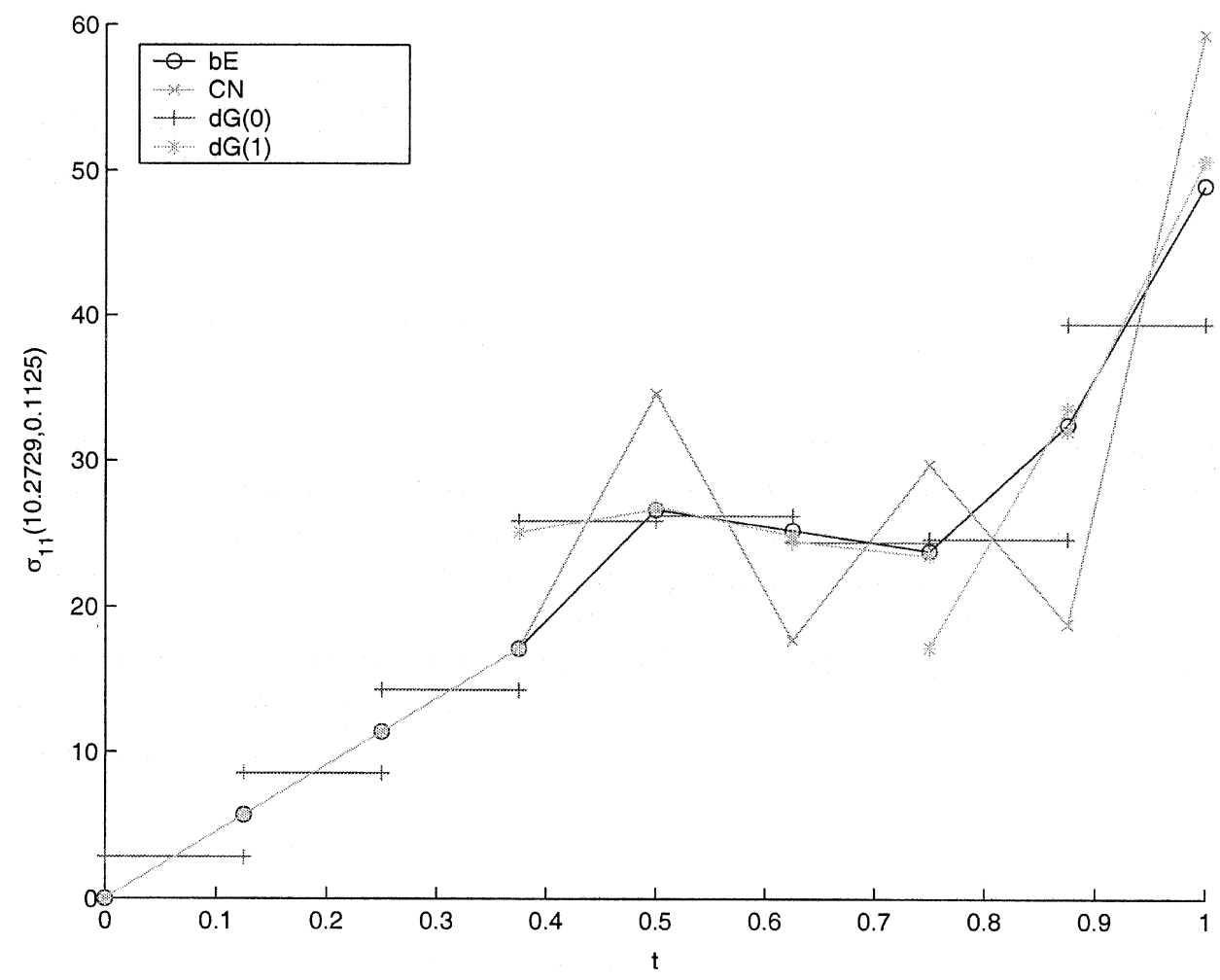

Fig. 1. History of discrete stress component $\sigma_{11}(10.2729,0.1125, t)$ at a point $\mathrm{A}^{\prime}$ as a function of time in the time interval $[0,1]$. 
numerical comparisons. A forthcoming paper [3] will explore the convergence behavior of the schemes from a theoretical and experimental point of view.

The rest of the paper is organized as follows. The strong and weak evolution problem are stated for a model in elastoplasticity in its primal and dual formulation in Section 2. The time discretization by generalized mid-point rules is explained in Section 3. The new discontinuous Galerkin time-discretization scheme is derived in Section 4 with emphasis on distributional time derivatives of discontinuous functions. The discrete conditions within each time step are not easily resolved and so Section 5 is concerned with solution algorithms for the implementation. Two quantitative examples are presented in Section 6 for a validation and illustration of the new schemes.

\section{Primal and dual formulation}

This section is devoted to the strong form of a model example in elastoplasticity with hardening and the weak primal and dual form [12]. The generalized stress and generalized plastic strains are given as

$$
\Sigma=(\sigma, \chi) \text { and } P=(p, \xi),
$$

respectively. The stress variable $\sigma$ and the total (linear Green) strain,

$$
\varepsilon(u):=\operatorname{sym} D u=\left(u_{j, k}+u_{k, j}\right) / 2 \quad j, k=1,2, \ldots, d,
$$

are linked with the irreversible plastic strain $p$ through an additive split

$$
\varepsilon(u)=\mathbb{C}^{-1} \sigma+p
$$

of small strain plasticity. The fourth-order elasticity tensor $\mathbb{C}$ acts as

$$
\mathbb{C} q=\lambda \operatorname{tr}(q) \mathbf{1}+2 \mu q \text { for all } q \in \mathbb{R}_{\mathrm{sym}}^{d \times d}
$$

with $\operatorname{trace} \operatorname{tr}(q):=q_{11}+\cdots+q_{d d}$, the $d \times d$ unit matrix $\mathbf{1}$, and the Lamé constants $\lambda, \mu>0$. The displacement field $u$ is supposed to satisfy Dirichlet boundary conditions in the form

$$
u=u_{\mathrm{D}} \quad \text { on } \Gamma_{\mathrm{D}}
$$

for a fixed closed part $\Gamma_{\mathrm{D}}$ of $\partial \Omega=\Gamma$ of positive $d-1$ dimensional (surface) measure. Equilibrium reads in local form

$$
\sigma=\sigma^{\mathrm{T}} \quad \text { and } \quad \operatorname{div} \sigma+f=0 \quad \text { in } \Omega
$$

plus Neumann boundary conditions

$$
\sigma n=g \quad \text { on } \Gamma_{\mathrm{N}}:=\Gamma \backslash \Gamma_{\mathrm{D}}
$$

on the remaining part of the boundary.

The internal energy assumes the form

$$
F(e, \xi):=1 / 2(e: \mathbb{C} e+\xi \cdot \mathbb{H} \xi)
$$

for the fourth-order elasticity tensor $\mathbb{C}$ and a symmetric and positive definite hardening tensor $\mathbb{Z}$. Internal (hardening) variables $\xi$ are written (symbolically) as $m$ dimensional vectors (e.g., the $m$ components of a symmetric $d \times d$ tensor for kinematic hardening or scalar, $m=1$, for linear isotropic hardening). Hence, $\xi$, $\chi \in \mathbb{R}^{m}$ and

$$
\mathbb{H} \in \mathbb{R}_{\mathrm{sym}}^{m \times m}
$$

is identified with a symmetric and positive definite $m \times m$ matrix. Then $\varepsilon(u)=e+p$ and

$$
\sigma=\partial F(e, \xi) / \partial e=\mathbb{C} e \text { and } \chi=-\partial F(e, \xi) / \partial \xi=-\mathbb{M}
$$


The Prandtl-Reuß, flow rule reads ( $\dot{p}$ denotes the time derivative of $p$ )

$$
\dot{p} \in N_{k}(\Sigma):=\left\{Q \in \mathbb{R}_{\mathrm{sym}}^{d \times d} \times \mathbb{R}^{m}: \forall T \in K, Q \star(T-\Sigma) \leqslant 0\right\},
$$

where the set of admissible generalized stresses $K \subset \mathbb{R}_{\mathrm{sym}}^{d \times d} \times \mathbb{R}^{m}$ is determined by the yield function (e.g., the von-Mises yield function) $\Phi: \mathbb{R}_{\mathrm{sym}}^{d \times d} \times \mathbb{R}^{m} \rightarrow \mathbb{R}$ via

$$
K:=\left\{T \in \mathbb{R}_{\mathrm{sym}}^{d \times d} \times \mathbb{R}^{m}: \Phi(T) \leqslant 0\right\} .
$$

Throughout this paper, we distinguish between the scalar products $\cdot$, :, $\star$, defined for vectors $u, v, d \times d$ matrices $p, q$, and generalized stresses or strains $P, Q$ by $u \cdot v=u_{1} v_{1}+\cdots+u_{d} v_{d}, p: q:=\sum_{j, k=1}^{d} p_{j k} q_{j k}$, and $P \star Q=(p, \xi) \star(q, \chi)=p: q+\xi \cdot \chi$.

Then, given data $u_{\mathrm{D}}, f$, and $g$ as functions in time $[0, T]$ and space, given consistent homogeneous initial conditions (i.e., $u_{\mathrm{D}}=0=f=g$ for $t=0$ ) the elastoplastic time evolution determines $u, \sigma, \chi, p$ and $\xi$ as functions on $[0, T] \times \Omega$ with

$$
\sigma=\sigma^{\mathrm{T}}=\mathbb{C}(\varepsilon(u)-p), \quad \operatorname{div} \sigma+f=0, \quad(\dot{p}, \dot{\xi}) \in N_{K}(\sigma, \chi) \quad \text { in }[0, T] \times \Omega
$$

and the boundary conditions

$$
u=u_{\mathrm{D}} \text { on }[0, T] \times \Gamma_{\mathrm{D}} \text { and } \quad \sigma n=g \text { on }[0, T] \times \Gamma_{\mathrm{N}} .
$$

Following [6,12] the primal and dual formulation differ in the treatment of the elastoplastic evolution law. Convex analysis $[10,22]$ reveals that the above formulation allows an equivalent reformulation via

$$
\dot{P} \in N_{K}(\Sigma) \Longleftrightarrow \Sigma \in \partial \operatorname{supp}_{K}(\dot{P}) .
$$

The first inclusion is defined above and, given $K$ via a yield function $\Phi$, reads

$$
\Phi(\Sigma) \leqslant 0 \text { and for all } T \in \mathbb{R}_{\mathrm{sym}}^{d \times d} \times \mathbb{R}^{m} \text { with } \Phi(T) \leqslant 0 \text {, there holds } \dot{P} \star(T-\Sigma) \leqslant 0 .
$$

The second inclusion involves the support function

$$
\operatorname{supp}_{K}(Q):=\sup _{T \in K} Q \star T=\sup _{\Phi(T) \leqslant 0} Q \star T
$$

and its subdifferential $\partial \operatorname{supp}_{K}$, e.g.,

$$
\Sigma \in \partial \operatorname{supp}_{K}(\dot{P}) \Longleftrightarrow \Sigma(Q-\dot{P}) \leqslant \operatorname{supp}_{K}(Q)-\operatorname{supp}_{K}(\dot{P}) \quad \text { for all } Q \in \mathbb{R}_{\mathrm{sym}}^{d \times d} \times \mathbb{R}^{m}
$$

The corresponding weak formulations are derived by a principle of virtual displacements or by testing with a test function. The two resulting variational inequalities are summarized below; we refer to [4,12] for further details.

Primal formulation: Seek $(u, p, \xi):[0, T] \rightarrow \mathbb{R}^{d} \times \mathbb{R}_{\mathrm{sym}}^{d \times d} \times \mathbb{R}^{m}$ with homogeneous initial values and, for almost every time $t \in(0, T)$ there holds

$$
\begin{aligned}
& \int_{\Omega} \mathbb{C}(\varepsilon(u(t))-p(t)):(\varepsilon(v)-\dot{p}(t)+q) \mathrm{d} x-\int_{\Omega} \xi(t) \cdot \mathbb{H}(\zeta-\dot{\xi}(t)) \mathrm{d} x \\
& \quad \leqslant \int_{\Omega} f(t) \cdot v \mathrm{~d} x+\int_{\Gamma_{\mathrm{D}}} g(t) \cdot v \mathrm{~d} s+\int_{\Omega} \operatorname{supp}_{K}(q, \zeta) \mathrm{d} x-\int_{\Omega} \operatorname{supp}_{K}(\dot{p}(t), \dot{\xi}(t)) \mathrm{d} x
\end{aligned}
$$

for all $v \in H_{\mathrm{D}}^{1}(\Omega):=\left\{w \in H^{1}(\Omega)^{d}: w=0\right.$ on $\left.\Gamma_{\mathrm{D}}\right\}$ and all $(q, \zeta) \in L^{2}\left(\Omega ; \mathbb{R}_{\mathrm{sym}}^{d \times d} \times \mathbb{R}^{m}\right)$ plus the Dirichlet boundary condition $u(t)=u_{\mathrm{D}}(t)$ on $\Gamma_{\mathrm{D}}$. 
The test function spaces are based on standard Lebesgue and Sobolev spaces, respectively,

$$
\begin{aligned}
& L^{2}(\Omega):=\left\{v: \Omega \rightarrow \mathbb{R}: v \text { measurable with } \int_{\Omega}|v|^{2} \mathrm{~d} x<\infty\right\} \\
& H^{1}(\Omega):=\left\{v \in L^{2}(\Omega): \forall j=1, \ldots, d, \partial v / \partial x_{j} \in L^{2}(\Omega)\right\}
\end{aligned}
$$

and powers thereof (i.e., all components belong to the respective space); $\partial v / \partial x_{j}$ is a weak derivative $[9,22]$.

Dual formulation: Seek $(u, \sigma, \chi):[0, T] \times \Omega \rightarrow \mathbb{R}^{d} \times \mathbb{R}_{\mathrm{sym}}^{d \times d} \times \mathbb{R}^{m}$ with homogeneous initial values and, for almost every time $t \in(0, T)$, there holds

$$
\int_{\Omega} \sigma(t): \varepsilon(v) \mathrm{d} x=\int_{\Omega} f(t) \cdot v \mathrm{~d} x+\int_{\Gamma_{T}} g(t) \cdot v \mathrm{~d} s
$$

for all $v \in H_{\mathrm{D}}^{1}(\Omega)$ and $\Phi(\sigma(t), \chi(t)) \leqslant 0$ and, for all $(\tau, \psi) \in L^{2}\left(\Omega ; \mathbb{R}_{\mathrm{sym}}^{d \times d} \times \mathbb{R}^{m}\right)$ with $\Phi(\tau, \psi) \leqslant 0$ there holds

$$
\int_{\Omega}\left(\varepsilon\left(\dot{u}(t)-\mathbb{C}^{-1} \dot{\sigma}(t)\right)\right):(\tau-\sigma(t)) \mathrm{d} x-\int_{\Omega} \dot{\chi}(t) \star \mathbb{Q}^{-1}(\psi-\chi(t)) \mathrm{d} x \leqslant 0
$$

plus the Dirichlet boundary condition $u(t)=u_{\mathrm{D}}$ on $\Gamma_{\mathrm{D}}$.

Remark 2.1. Dual and primal formulation are, on the continuous level, equivalent $[4,12]$. The role of the time derivative as well as the choice of the (main) variables are different.

\section{Discretization}

This section is devoted to generalized mid-point time discretization and finite element space discretization of the primal and dual formulation of the elastoplastic model problem. Within the framework of Section 2 we describe a general-time difference scheme that includes the bE and the CN scheme for $\Theta=1$ and $\Theta=1 / 2$, respectively.

The time interval $(0, T]$ is partitioned in $L$ subintervals $I_{j}=\left(t_{j-1}, t_{j}\right]$ (open at the left and closed at the right), $j=1, \ldots, L$, according to

$$
t_{0}=0<t_{1}<t_{2}<\cdots<t_{L}=T ; \quad \mathscr{I}=\left\{I_{1}, I_{2}, \ldots, I_{L}\right\} .
$$

Given discrete data $x_{0}, x_{1}, \ldots, x_{L}$ the associated piecewise affine and globally continuous interpolant $\tilde{x}$ is given by

$$
\tilde{x}(t)=\left(t_{j}-t\right) / k_{j} x_{j-1}+\left(t-t_{j-1}\right) / k_{j} x_{j} \text { for } t_{j-1}<t \leqslant t_{j}=t_{j-1}+k_{j} .
$$

We write $\tilde{x} \in \mathscr{S}^{1}(\mathscr{I} ; X)$ for $\mathscr{I}$ piecewise affine and globally continuous $X$-valued functions. Notice that $\tilde{x}$ has a derivative on $I_{j}$, namely $\dot{\tilde{x}}(t)=\left(x_{j}-x_{j-1}\right) / k_{j}$ for $t \in I_{j}$. (Here, we follow the convention that $\dot{\tilde{x}}\left(t_{j}\right)$ equals its left-sided time derivative.) The idea of finite difference schemes (in time) is to replace derivatives, e.g., $(\dot{p}(t), \dot{\xi}(t))$ or $(\dot{u}(t), \dot{\sigma}(t), \dot{\chi}(t))$, by the discrete time derivatives, e.g., $\left(\left(p\left(t_{j}\right)-p\left(t_{j-1}\right)\right) / k_{j},\left(\xi\left(t_{j}\right)-\xi\left(t_{j-1}\right)\right) / k_{j}\right)$ or $\left(\left(u\left(t_{j}\right)-u\left(t_{j-1}\right)\right) / k_{j},\left(\sigma\left(t_{j}\right)-\sigma\left(t_{j-1}\right)\right)\right) / k_{j},\left(\left(\chi\left(t_{j}\right)-\chi\left(t_{j-1}\right)\right) / k_{j}\right)$, and replace the evaluation at time $t$ by the evaluation at a mid-point $t_{j-1}+\Theta k_{j}$ for some $\Theta, 0<\Theta \leqslant 1$. Before we state the resulting identities for the primal and dual formulation, we will specify the space discretization.

The domain $\Omega$ is partitioned into triangles, parallelograms for 2D and tetrahedra for $3 \mathrm{D}$. The resulting triangulation $\mathscr{T}$ is supposed to be regular in the sense of Ciarlet [5,8]. For each element $T, P_{k}(T)$ denotes the algebraic polynomials on $T$ of total degree $\leqslant k$ if $T$ is a triangle or tetrahedron or of partial degree $\leqslant k$ if $T$ is a parallelogram. Then, the required finite element function spaces read 


$$
\begin{aligned}
& \mathscr{L}^{k}(\mathscr{T}):=\left\{v \in L^{2}(\Omega): \forall T \in \mathscr{T},\left.v\right|_{T} \in P_{k}(T)\right\}, \\
& \mathscr{S}^{1}(\mathscr{T}):=\left(\mathscr{L}^{1}(\mathscr{T} \cap C(\bar{\Omega}))^{d},\right. \\
& \mathscr{S}_{\mathrm{D}}^{1}(\mathscr{T}):=H_{\mathrm{D}}^{1}(\Omega) \cap \mathscr{S}^{1}(\mathscr{T}), \\
& \mathbb{L}(\mathscr{T}):=\left\{(p, \xi) \in L^{2}\left(\Omega ; \mathbb{R}_{\mathrm{sym}}^{d \times d} \times \mathbb{R}^{m}\right): p_{j k}, \xi_{\ell} \in \mathscr{L}^{0}(\mathscr{T})\right\}=\mathscr{L}^{0}\left(\mathscr{T} ; \mathbb{R}_{\mathrm{sym}}^{d \times d}\right) \times \mathscr{L}^{0}\left(\mathscr{T} ; \mathbb{R}^{m}\right) .
\end{aligned}
$$

The finite element approximation is denoted by a subindex $h$ (which is neglected for its continuous counterpart) although the underlying discretization is based on a partition $\mathscr{I}$ in time and $\mathscr{T}$ in space. The substitution of the continuous functions by their discrete approximations in the finite difference scheme leads to the following two discrete generalized mid-point difference schemes. Set $u_{h, 0}, p_{h, 0}, \xi_{h, 0}, \sigma_{h, 0}, \chi_{h, 0}$ equal to zero.

$\Theta$-discrete-primal formulation: Given $\left(u_{h, j-1}, p_{h, j-1}, \xi_{h, j-1}\right)$ and $j=1,2, \ldots, L-1$, seek $u_{h, j} \in u_{\mathrm{D}, h, j}+$ $\mathscr{S}_{\mathrm{D}}^{1}(\mathscr{T})$ and $\left(p_{h, j}, \xi_{h, j}\right) \in \mathbb{L}(\mathscr{T})$ with

$$
\begin{aligned}
\int_{\Omega} \mathbb{C} & \left(\varepsilon\left(u_{h, j-1+\Theta}\right)-p_{h, j-1+\Theta}\right):\left(\varepsilon\left(v_{h}\right)-\left(p_{h, j}-p_{h, j-1}\right) / k_{j}+q_{h}\right) \mathrm{d} x \\
& -\int_{\Omega} \xi_{h, j-1+\Theta} \cdot \mathbb{U}\left(\zeta_{h}-\left(\xi_{h, j}-\xi_{h, j-1}\right) / k_{j}\right) \mathrm{d} x \\
\leqslant & \int_{\Omega} f\left(t_{j-1+\Theta}\right) \cdot v_{h} \mathrm{~d} x+\int_{\Gamma_{\mathrm{N}}} g\left(t_{j-1+\Theta}\right) \cdot v_{h} \mathrm{~d} s+\int_{\Omega} \operatorname{supp}_{K}\left(q_{h}, \zeta_{h}\right) \mathrm{d} x \\
& -\int_{\Omega} \operatorname{supp}_{K}\left(\left(p_{h, j}-p_{h, j-1}\right) / k_{j},\left(\xi_{h, j}-\xi_{h, j-1}\right) / k_{j}\right) \mathrm{d} x
\end{aligned}
$$

for all $v_{h} \in \mathscr{S}_{\mathrm{D}}^{1}(\Omega)$ and all $\left(q_{h}, \zeta_{h}\right) \in \mathbb{L}(\mathscr{T})$; here, $u_{h, j-1+\Theta}, p_{h, j-1+\Theta}, \xi_{h, j-1+\Theta}$ abbreviate

$$
(1-\Theta) u_{h, j-1}+\Theta u_{h, j}, \quad(1-\Theta) p_{h, j-1}+\Theta p_{h, j}, \quad(1-\Theta) \xi_{h, j-1}+\Theta \xi_{h, j}
$$

i.e., $\tilde{u}_{h}\left(t_{j-1+\Theta}\right), \tilde{p}_{h}\left(t_{j-1+\Theta}\right), \tilde{\xi}\left(t_{j-1+\Theta}\right)$, respectively.

$\Theta$-discrete dual formulation: Given $\left(u_{h, j-1}, \sigma_{h, j-1}, \chi_{h, j-1}\right)$ and $j=1,2, \ldots, L-1$, seek $u_{h, j} \in u_{\mathrm{D}, h, j}+\mathscr{S}_{\mathrm{D}}^{1}(\mathscr{T})$ and $\left(\sigma_{h, j}, \chi_{h, j}\right) \in \mathbb{L}(\mathscr{T})$ with

$$
\int_{\Omega} \sigma_{h, j-1+\Theta}: \varepsilon\left(v_{h}\right) \mathrm{d} x=\int_{\Omega} f\left(t_{j-1+\Theta}\right) \cdot v_{h} \mathrm{~d} x+\int_{\Gamma_{\mathrm{N}}} g\left(t_{j-1+\Theta}\right) \cdot v_{h} \mathrm{~d} s
$$

for all $v_{h} \in \mathscr{S}_{\mathrm{D}}^{1}(\mathscr{T})$ and $\Phi\left(\sigma_{h, j}, \chi_{h, j}\right) \leqslant 0$ and, for all $\left(\tau_{h}, \psi_{h}\right) \in \mathbb{L}(\mathscr{T})$ with $\Phi\left(\tau_{h}, \psi_{h}\right) \leqslant 0$ there holds

$$
\begin{gathered}
\int_{\Omega}\left(\varepsilon\left(\left(u_{h, j}-u_{h, j-1}\right) / k_{j}\right)-\mathbb{C}^{-1}\left(\sigma_{h, j}-\sigma_{h, j-1}\right) / k_{j}\right):\left(\tau_{h}-\sigma_{h, j-1+\Theta}\right) \mathrm{d} x \\
-\int_{\Omega}\left(\chi_{h, j}-\chi_{h, j-1}\right) / k_{j} \cdot \mathbb{Q}^{-1}\left(\psi_{h}-\chi_{h, j-1+\Theta}\right) \mathrm{d} x \leqslant 0 .
\end{gathered}
$$

Again, $u_{h, j-1+\Theta}, \sigma_{h, j-1+\Theta}, \chi_{h, j-1+\Theta}$ abbreviates

$$
(1-\Theta) u_{h, j-1}+\Theta u_{h, j}, \quad(1-\Theta) \sigma_{h, j-1}+\Theta \sigma_{h, j}, \quad(1-\Theta) \chi_{h, j-1}+\Theta \chi_{h, j},
$$

i.e., $\tilde{u}_{h}\left(t_{j-1+\Theta}\right), \tilde{\sigma}_{h}\left(t_{j-1+\Theta}\right), \tilde{\chi}\left(t_{j-1+\Theta}\right)$, respectively.

Remark 3.1. Recall that the most prominent versions are the Crank-Nicholson time discretization (CN for short) for $\Theta=1 / 2$ and backward Euler scheme (abbreviated bE) for $\Theta=1[12,20]$. 
Remark 3.2. The above described numerical scheme is simplified in that the time-evaluation point $t_{j-1+\Theta}$ is described by one global parameter and the finite element spaces are fixed in each time step. The more general situation for flexible time steps and different $\Theta$ for different time steps can be easily extrapolated from the given descriptions. The presentation of the $\Theta$-discrete finite difference scheme suffices to model the numerical examples of Section 6.

Remark 3.3. The implementation and convergence analysis of the $\Theta$-discrete finite difference schemes may be found in $[2,12-15,20]$; particular attention to the mesh-design within one time step is paid in $[4,6,7,11,16]$.

\section{Discontinuous Galerkin time discretization}

This section is devoted to the motivation and design of a new class of discontinuous Galerkin time discretizations (abbreviated $\mathrm{dG}$ and $\mathrm{dG}(k)$ for the $k$ th-order $\mathrm{dG}$ scheme) of elastoplastic evolution problems. The point of departure is the concept of a distributional derivative of discontinuous $\mathscr{I}$-piecewise smooth functions.

Example 4.1 (distributional derivative of Heaviside function). A well-known result in the theory of distributions $\mathscr{D}^{\prime}$ reads

$$
H^{\prime}=\delta \text { in } \mathscr{D}^{\prime}
$$

and means: For any $\varphi \in \mathscr{D}(\mathbb{R})$, i.e., $\varphi$ is $\mathscr{C}^{\infty}$ and has compact support in $\mathbb{R}$, there holds

$$
-\int_{\mathbb{R}} H(t) \varphi^{\prime}(t) \mathrm{d} x=\varphi(0)=:\langle\delta, \varphi\rangle
$$

for the Heaviside function $H(t):=0$ for $t \leqslant 0$ and $H(t)=1$ for $t>0$ and Dirac's delta distribution $\delta$ (which acts by taking the value of the test function at zero). The proof is by integration by parts (or fundamental theorem of calculus) on a large interval $(-\ell,+\ell)$ such that $\varphi$ vanishes outside $(-\ell,+\ell)$. Then,

$$
-\int_{\mathbb{R}} H(t) \varphi^{\prime}(t) \mathrm{d} t=-\int_{0}^{\ell} \varphi^{\prime}(t) \mathrm{d} t=-\varphi(\ell)+\varphi(0)=\varphi(0)
$$

(since $\varphi(\ell)=0$ for sufficient large $\ell$ ).

Example 4.2 (distributional derivative of $\mathscr{I}$-piecewise smooth functions). Let $\mathscr{I}$ denote the partition of the time domain $(0, T]$ introduced in the previous section. Let $u \in \mathscr{C}^{1}(\mathscr{I})$ denote the set of all functions $u \in L^{\infty}(\mathbb{R})$ with $\left.u\right|_{I_{j}} \in \mathscr{C}^{1}\left[t_{j-1}, t_{j}\right]$ and constants $\left.u\right|_{(-\infty, 0]}:=u\left(0^{-}\right)$and $\left.u\right|_{(T, \infty)}:=u\left(T^{+}\right)$outside $(0, T]$. For such $u$, the one-sided limits exists,

$$
\lim _{t \rightarrow t_{j}^{ \pm}} u(t)=: u\left(t_{j}^{ \pm}\right)
$$

i.e., $\lim _{t \rightarrow t_{j}^{+}} u(t)=\lim _{I_{j+1} \ni t \rightarrow t_{j}} u(t)$ and $\lim _{t \rightarrow t_{j}^{-}} u(t)=\lim _{I_{j} \ni t \rightarrow t_{j}} u(t)$ and $I_{0}:=(-\infty, 0], I_{L+1}:=(T, \infty)$, and we may define the jumps

$$
[u]_{j}:=u\left(t_{j}^{+}\right)-u\left(t_{j}^{-}\right) \text {for } j=0,1,2, \ldots, L .
$$

Finally, since $\left.u\right|_{\left(t_{j-1}, t_{j}\right)}$ is $\mathscr{C}^{1}$ there exists $u_{\tau}:=u^{\prime}$ on each $\left(t_{j-1}, t_{j}\right)$. The composition $u_{\tau}$ is the $\mathscr{I}$ piecewise time derivative of $u,\left.u_{\tau}\right|_{I_{j}}=\left.u^{\prime}\right|_{t_{j-1}, t_{j}}$ for $j=0,1, \ldots, L$, which vanishes outside $(0, T)$. Then, the distributional derivative $\dot{u}$ is defined by 


$$
\int_{\mathbb{R}} \dot{u}(t) v(t) \mathrm{d} t=-\int_{\mathbb{R}} u(t) \dot{v}(t) \mathrm{d} t \quad \text { for all } v \in \mathscr{D}(\mathbb{R})
$$

and the point is that the right-hand side is well-understood since $\dot{v}$ is smooth. In abstract terms, the distributional derivative $\dot{u}$ is the sum of the piecewise contribution $u_{\tau}$ and, owing to the preceding example, the jump on each $t_{j}$, namely,

$$
\dot{u}=u_{\tau}+\sum_{j=0}^{L}[u]_{j} \delta_{t_{j}} \quad \text { in } \mathscr{D}^{\prime}(\mathbb{R}) .
$$

Here $[u]_{j}$ is a scalar factor and $\delta_{t_{j}}$ is the delta distribution supported at $t_{j}$, i.e., $\left\langle\delta_{t_{j}}, v\right\rangle=v\left(t_{j}\right)$. This means that, for any $v \in \mathscr{D}(\mathbb{R})$,

$$
\int_{\mathbb{R}} \dot{u}(t) v(t) \mathrm{d} t=\int_{\mathbb{R}} u_{\tau}(t) v(t) \mathrm{d} t+\sum_{j=0}^{L}[u]_{j} v\left(t_{j}\right) .
$$

The proof follows by the linearity of the distributional derivative and the jump identity in the preceding example. A direct proof via an integration by parts is left to the reader.

In the example, jumps and one-sided limits are written for real-valued functions; the notation is adopted for Lebesgue- and Sobolev-functions as well.

The two preliminary examples describe the action of $\dot{u}$ for $u \in \mathscr{C}^{1}(\mathscr{I})$ onto continuous test functions. A $\mathrm{dG}$ scheme allows discontinuous test functions for which we establish a proper meaning of $\dot{u}$. The starting point focuses on one fixed interval $I_{j}$. Given $v \in \mathscr{C}^{1}(\mathscr{I})$ with $v=0$ outside $I_{j}$ we define a globally continuous and piecewise $\mathscr{C}^{1}$ function $v_{\varepsilon}$ by $v_{\varepsilon}(t):=\chi_{\varepsilon}^{j}(t) v(t)$ with

$$
\chi_{\varepsilon}^{j}(t):= \begin{cases}\left(t-t_{j-1}\right) / \varepsilon+1 & \text { for } t_{j-1}-\varepsilon \leqslant t \leqslant t_{j-1}, \\ 1 & \text { for } t_{j-1} \leqslant t \leqslant t_{j}-\varepsilon, \\ \left(t_{j}-t\right) / \varepsilon & \text { for } t_{j}-\varepsilon \leqslant t \leqslant t_{j}, \\ 0 & \text { elsewhere. }\end{cases}
$$

Remark 4.1. Here we suppose that $\left.v\right|_{I_{j}} \in \mathscr{C}^{1}\left[t_{j-1}, t_{j}\right]$ can be extended to the left onto $v \in \mathscr{C}^{1}\left[t_{j-1}-\varepsilon, t_{j}\right]$. This is no restriction for the polynomial test function $v$ in the discrete scheme. The final result will be independent of this $\mathscr{C}^{1}$ extension.

The evaluation of $\int_{\mathbb{R}} \dot{u}(t) v_{\varepsilon}(t) \mathrm{d} t$ for $u \in \mathscr{C}^{1}(\mathscr{I})$ and the test function $v_{\mathscr{E}}$ follows with the above formula and reads

$$
\int_{\mathbb{R}} \dot{u}(t) v_{\varepsilon}(t) \mathrm{d} t=\int_{t_{j-1}-\varepsilon}^{t_{j}} u_{\tau}(t) v_{\varepsilon}(t) \mathrm{d} t+[u]_{j-1} v_{\varepsilon}\left(t_{j-1}\right) .
$$

Here we used $v_{\varepsilon}\left(t_{j}\right)=0$ and the continuity of $v_{\varepsilon}$ at $t_{j-1}$. The right-hand side is analyzed in the limit $\varepsilon \rightarrow 0$. Since $u_{\tau} v_{\varepsilon}$ is bounded, the first term yields

$$
\lim _{\varepsilon \rightarrow 0} \int_{t_{j-1}-\varepsilon}^{t_{j}} u_{\tau}(t) v_{\varepsilon}(t) \mathrm{d} t=\int_{I_{j}} u_{\tau}(t) v(t) \mathrm{d} t .
$$

Since $\chi_{\varepsilon}^{j}\left(t_{j-1}\right)=1$, the second term equals

$$
[u]_{j-1} v_{\varepsilon}\left(t_{j-1}\right)=[u]_{j-1} v\left(t_{j-1}^{+}\right)
$$

(the value $v\left(t_{j-1}^{-}\right)$is zero and we extended $v$ onto $\left(t_{j-1}-\varepsilon, t_{j}\right)$ around $t_{j-1}$ with this value $v\left(t_{j-1}^{+}\right)$). 
Up to this point we were concerned with one time interval $I_{j}$ and obtained

$$
\lim _{\varepsilon \rightarrow 0} \int_{\mathbb{R}} \dot{u}(t) v_{\varepsilon}(t) \mathrm{d} t=\int_{I_{j}} u_{\tau}(t) v(t) \mathrm{d} t+[u]_{j} v_{\varepsilon}\left(t_{j-1}^{+}\right) .
$$

The same procedure for a general $v \in \mathscr{C}^{1}(\mathscr{I})$ with the test function $v_{\varepsilon}^{j}:=\left(\right.$ extension of $\left.\left.v\right|_{I_{j}} \chi_{\varepsilon}^{j}\right)$ and their sum $v_{\varepsilon}$ results into

$$
\lim _{\varepsilon \rightarrow 0} \int_{\mathbb{R}} \dot{u}\left(\sum_{j=1}^{L} v_{\varepsilon}^{j}\right) \mathrm{d} t=\int_{0}^{T} u_{\tau}(t) v(t) \mathrm{d} t+\sum_{j=1}^{L}[u]_{j-1} v\left(t_{j-1}^{+}\right) .
$$

The right-hand side makes sense for $u, v \in \mathscr{C}^{1}(\mathscr{I})$ and shall be employed to replace the derivative $\dot{u}$ evaluated for the test function $v$.

Remark 4.2. Since, for a continuous test function $v \in \mathscr{C}^{1}(\mathbb{R}) \subset \mathscr{C}^{1}(\mathscr{I})$, the last right-hand side coincides with the distributional derivative $\dot{u}$ tested with $v$, the last expression is one generalization of the distributional time derivative of $u$.

Remark 4.3. Given $u \in \mathscr{C}^{1}(\mathscr{I})$, there are various different ways of generalizations of the expression $\int_{\mathbb{R}} \dot{u}(t) v(t) \mathrm{d} t$ for discontinuous $v$. For example, the choices

$$
\chi_{\varepsilon}^{j}(t):=\left\{\begin{array}{ll}
\left(t-t_{j-1}\right) / \varepsilon & \text { for } t_{j-1} \leqslant t \leqslant t_{j-1}+\varepsilon, \\
1 & \text { for } t_{j-1}+\varepsilon \leqslant t \leqslant t_{j}-\varepsilon, \\
\left(t_{j}-t\right) / \varepsilon & \text { for } t_{j}-\varepsilon \leqslant t \leqslant t_{j}, \\
0 & \text { elsewhere }
\end{array} \text { or } \quad \chi_{\varepsilon}^{j}(t):= \begin{cases}\left(t-t_{j-1}\right) / \varepsilon & \text { for } t_{j-1} \leqslant t \leqslant t_{j-1}+\varepsilon, \\
1 & \text { for } t_{j-1}+\varepsilon \leqslant t \leqslant t_{j}, \\
\left(t_{j}-t\right) / \varepsilon+1 & \text { for } t_{j} \leqslant t \leqslant t_{j}+\varepsilon, \\
0 & \text { elsewhere }\end{cases}\right.
$$

lead to different formulae. The first case misses the jump contributions and the second (with $v$ extended continuously to $\left(t_{j-1}, t_{j}+\varepsilon\right)$ ) yields a forward formula with the jumps $[u]_{j} v\left(t_{j}^{-}\right)$. The objection against the first choice of $\chi_{\varepsilon}^{j}$ is that it yields no generalization of $\int_{\mathbb{R}} \dot{u}(t) v(t) \mathrm{d} t$ for continuous $v$. The objection against the second choice is simply that is does not lead to a single-step method in the end. (This latter outcome will become transparent in the next section.)

We are now in the position to state the $\mathrm{dG}(k)$ methods, $k=0,1,2, \ldots$, where the discrete solution and the test function belong to $\mathscr{L}^{k}\left(\mathscr{I} ; \mathscr{S}^{1}(\mathscr{T})^{d} \times \mathbb{L}(\mathscr{T})\right)$,

$$
\begin{aligned}
& \mathscr{L}^{k}(\mathscr{I} ; X):=\left\{v:[0, T] \rightarrow X: \forall I_{j} \in \mathscr{I},\left.v\right|_{I_{j}} \in \mathscr{P}_{k}\left(I_{j} ; X\right)\right\}, \\
& \mathscr{P}_{k}\left(I_{j} ; X\right):=\left\{v: I_{j} \rightarrow X: \exists v_{0}, v_{1}, \ldots, v_{k} \in X \forall t \in I_{j}, v(t)=v_{0}+v_{1} t+\cdots+v_{k} t^{k}\right\} .
\end{aligned}
$$

$d G(k)$-discrete dual formulation: Seek $\left(u_{h}, \Sigma_{h}\right) \in \mathscr{L}^{k}\left(\mathscr{I} ; \mathscr{S}^{1}(\mathscr{T})^{d} \times \mathbb{L}(\mathscr{T})\right), \Sigma_{h}=\left(\sigma_{h}, \chi_{h}\right)$, with $\left(u_{h}, \sigma_{h}\right.$, $\left.\chi_{h}\right)(t)=0$ for $t \leqslant 0, u_{h}(t)=u_{\mathrm{D}, h}(t)$ on $\Gamma_{\mathrm{D}}$ and $\Phi\left(\sigma_{h}, \chi_{h}\right)(t) \leqslant 0$ for $0 \leqslant t \leqslant T$; furthermore there holds

$$
\int_{0}^{T} \int_{\Omega} \sigma_{h}: \varepsilon\left(v_{h}\right) \mathrm{d} x \mathrm{~d} t=\int_{0}^{T} \int_{\Omega} f \cdot v_{h} \mathrm{~d} x \mathrm{~d} t+\int_{0}^{T} \int_{\Gamma_{\mathrm{N}}} g \cdot v_{h} \mathrm{~d} s \mathrm{~d} t
$$

for all $v_{h} \in \mathscr{L}^{k}\left(\mathscr{I} ; \mathscr{S}_{\mathrm{D}}^{1}(\mathscr{T})\right)$ and, for all $T_{h}:=\left(\tau_{h}, \psi_{h}\right) \in \mathscr{L}^{k}(\mathscr{I} ; \mathbb{L}(\mathscr{T}))$ with $\Phi\left(\tau_{h}, \psi_{h}\right)(t) \leqslant 0$ for $0 \leqslant t \leqslant T$, there holds

$$
\int_{0}^{T} \int_{\Omega} P_{h, \tau} \star\left(T_{h}-\Sigma_{h}\right) \mathrm{d} x \mathrm{~d} t+\sum_{j=1}^{L} \int_{\Omega}\left[P_{h}\right]_{j-1} \star\left(T_{h}-\Sigma_{h}\right)\left(t_{j-1}^{+}\right) \mathrm{d} x \leqslant 0,
$$

where $P_{h}:=\left(\varepsilon\left(u_{h}\right)-\mathbb{C}^{-1} \sigma_{h},-\mathbb{M}^{-1} \chi_{h}\right) \in \mathscr{L}^{k}(\mathscr{I} ; \mathbb{L}(\mathscr{T}))$.

The $\mathrm{dG}(k)$ discrete dual formulation is straight-forward in that a time derivative $\dot{P}_{h} \star\left(T_{h}-\Sigma_{h}\right)$ is substituted by the aforementioned formula. The corresponding situation is rather more involved for the primal 
formulation where, in addition, the term $\operatorname{supp}_{k}\left(\dot{P}_{h}\right)$ requires a discrete formulation. The difficulty is that the function $\operatorname{supp}_{K}$ (think of it as a modulus function) does not commute with time derivatives and is, in general, not even differentiable.

Fortunately, $\operatorname{supp}_{K}\left(P_{h}\right)$ is not an arbitrary function, but related to the set of admissible generalized stresses $K$ via a dual pairing. We employ

$$
\operatorname{supp}_{K}\left(\dot{P}_{h}\right)=\sup _{R \in K} R \star \dot{P}_{h}
$$

in the time-domain integral

$$
\int_{0}^{T} \int_{\Omega} \operatorname{supp}_{K}\left(\dot{P}_{h}\right) \mathrm{d} x \mathrm{~d} t=\int_{0}^{T} \int_{\Omega} \sup _{R \in K} R \star \dot{P}_{h} \mathrm{~d} x \mathrm{~d} t
$$

In the discrete setting, we replace the last integral by

$$
\sup _{R_{h} \in \mathscr{L}^{k}(\mathscr{F} ;[(\mathscr{T}))} \int_{0}^{T} \int_{\Omega} R_{h} \star \dot{P}_{h} \mathrm{~d} x \mathrm{~d} t
$$

and evaluate the aforementioned formula $\dot{P}_{h}=P_{h, \tau}+\sum_{j=1}^{L} \delta_{t_{j-1}^{+}}\left[P_{h}\right]_{j-1}$ for $R_{h}$. This motivates the interpretation of $\int_{0}^{T} \int_{\Omega} \operatorname{supp}_{K}\left(\dot{P}_{h}\right) \mathrm{d} x \mathrm{~d} t$ in the discretization as

$$
\sup _{R_{h} \in \mathscr{L}^{k}(\mathscr{F} ; \mathbb{L}(\mathscr{T}))}\left(\int_{0}^{T} \int_{\Omega} R_{h} \star P_{h, \tau} \mathrm{d} x \mathrm{~d} t+\sum_{j=1}^{L} \int_{\Omega} R_{h}\left(t_{j-1}^{+}\right) \star\left[P_{h}\right]_{j-1} \mathrm{~d} x\right)
$$

and results in the following discrete scheme.

$d G(k)$-discrete-primal formulation: Seek $\quad\left(u_{h}, P_{h}\right) \in \mathscr{L}^{k}\left(\mathscr{I} ; \mathscr{S}^{1}(\mathscr{T})^{d} \times \mathbb{L}(\mathscr{T})\right), \quad P_{h}=\left(p_{h}, \xi_{h}\right), \quad$ with $\left(u_{h}, p_{h}, \xi_{h}\right)(t)=0$ for $t \leqslant 0, u_{h}(t)=u_{\mathrm{D}, h}(t)$ on $\Gamma_{\mathrm{D}}$ for $0 \leqslant t \leqslant T$, and

$$
\begin{aligned}
\int_{0}^{T} \int_{\Omega} \mathbb{C}\left(\varepsilon\left(u_{h}\right)-p_{h}\right):\left(\varepsilon\left(v_{h}\right)-p_{h, \tau}+q_{h}\right) \mathrm{d} x \mathrm{~d} t-\int_{0}^{T} \int_{\Omega} \xi_{h} \cdot \mathbb{H}\left(\zeta_{h}-\xi_{h, \tau}\right) \mathrm{d} x \mathrm{~d} t \\
\quad-\sum_{j=1}^{L} \int_{\Omega} \mathbb{C}\left(\varepsilon\left(u_{h}\right)-p_{h}\right)\left(t_{j-1}^{+}\right):\left[p_{h}\right]_{j-1} \mathrm{~d} x+\sum_{j=1}^{L} \int_{\Omega} \xi_{h}\left(t_{j-1}^{+}\right) \cdot \mathbb{H}\left[\zeta_{h}\right]_{j-1} \mathrm{~d} x \\
\leqslant \int_{0}^{T} \int_{\Omega} f \cdot v_{h} \mathrm{~d} x \mathrm{~d} t+\int_{\Gamma_{\mathrm{N}}} g \cdot v_{h} \mathrm{~d} s \mathrm{~d} t+\sup _{R_{h} \in \mathscr{L}^{k}(\mathscr{T} ; \mathbb{L}(\mathscr{T}))} \int_{0}^{T} \int_{\Omega} R_{h} \star\left(q_{h}, \zeta_{h}\right) \mathrm{d} x \mathrm{~d} t \\
-\sup _{R_{h} \in \mathscr{L}^{k}(\mathscr{I} ; \mathbb{L}(\mathscr{T}))}\left(\int_{0}^{T} \int_{\Omega} R_{h} \star P_{h, \tau} \mathrm{d} x \mathrm{~d} t+\sum_{j=1}^{L} \int_{\Omega} R_{h}\left(t_{j-1}^{+}\right) \star\left[P_{h}\right]_{j-1} \mathrm{~d} x\right)
\end{aligned}
$$

for all $\left(v_{h},\left(q_{h}, \zeta_{h}\right)\right) \in \mathscr{L}^{k}\left(\mathscr{I} ; \mathscr{S}_{\mathrm{D}}^{1}(\mathscr{T}) \times \mathbb{L}(\mathscr{T})\right)$.

Remark 4.4. On the continuous level one can prove

$$
\sup _{\Phi(R) \leqslant 0} \int_{0}^{T} \int_{\Omega} R(t) \star Q(t) \mathrm{d} x \mathrm{~d} t=\int_{0}^{T}\left(\sup _{\Phi(R) \leqslant 0} \int_{\Omega} R \star Q(t) \mathrm{d} x\right) \mathrm{d} t=\int_{0}^{T} \int_{\Omega}\left(\sup _{\Phi(R) \leqslant 0} R \times Q(x, t)\right) \mathrm{d} x \mathrm{~d} t .
$$

This justifies the replacement of (4.1) by (4.2).

The two discrete $\mathrm{dG}$ schemes are written in integral form and will be recast into single-step methods in the subsequent section. 
Remark 4.5. The derivation of the two $d G$ schemes appears heuristic. At some points, various different choices would have resulted in other methods. The mathematical justification of the schemes introduced in this section is two-fold. Firstly, this Part I will show that the methods are feasible, i.e., they lead to singlestep methods and there are algorithms to solve the discrete problems. Moreover, the schemes lead to reasonable results in two applications in Section 6. Secondly, the forthcoming paper [3] will provide an a priori error analysis with asymptotic convergence rates. Moreover, the theoretical results are verified experimentally in a test example. Then, after the analysis is established, the fact that the schemes are practical and convergent, finally justifies our particular choices made in this section.

\section{Numerical algorithms}

This section is devoted to single-step descriptions of the discrete $\mathrm{dG}(k)$ primal and dual formulation for $k=0$ and $k=1$. The situation for $k=0$ is very similar to the $\mathrm{bE}$ scheme.

$d G(0)$ single-step primal formulation: Given $\left(u_{h, j-1}, p_{h, j-1}, \xi_{h, j-1}\right)$ and $j=1,2, \ldots, L-1$, seek $u_{h, j} \in u_{\mathrm{D}, h, j}+$ $\mathscr{S}_{\mathrm{D}}^{1}(\mathscr{T})$ and $\left(p_{h, j}, \xi_{h, j}\right) \in \mathbb{L}(\mathscr{T})$ with

$$
\begin{aligned}
\int_{\Omega} & \mathbb{C}\left(\varepsilon\left(u_{h, j}\right)-p_{h, j}\right):\left(\varepsilon\left(v_{h}\right)-\left(p_{h, j}-p_{h, j-1}\right)+q_{h}\right) \mathrm{d} x-\int_{\Omega} \xi_{h, j} \cdot \mathbb{M}\left(\zeta_{h}-\left(\xi_{h, j}-\xi_{h, j-1}\right)\right) \mathrm{d} x \\
\leqslant & \int_{t_{j-1}}^{t_{j}} \int_{\Omega} f(t) \cdot v_{h} \mathrm{~d} x \mathrm{~d} t+\int_{t_{j-1}}^{t_{j}} \int_{\Gamma_{\mathrm{N}}} g(t) \cdot v_{h} \mathrm{~d} s \mathrm{~d} t+\int_{\Omega} \operatorname{supp}_{K}\left(q_{h}, \zeta_{h}\right) \mathrm{d} x \\
& \quad-\int_{\Omega} \operatorname{supp}_{K}\left(p_{h, j}-p_{h, j-1}, \xi_{h, j}-\xi_{h, j-1}\right) \mathrm{d} x
\end{aligned}
$$

for all $v_{h} \in \mathscr{S}_{\mathrm{D}}^{1}(\Omega)$ and all $\left(q_{h}, \zeta_{h}\right) \in \mathbb{L}(\mathscr{T})$.

$d G(0)$ single-step dual formulation: Given $\left(u_{h, j-1}, \sigma_{h, j-1}, \chi_{h, j-1}\right)$ and $j=1,2, \ldots, L-1$, seek $u_{h, j} \in u_{\mathrm{D}, h, j}+$ $\mathscr{S}_{\mathrm{D}}^{1}(\mathscr{T})$ and $\left(\sigma_{h, j}, \chi_{h, j}\right) \in \mathbb{L}(\mathscr{T})$ with

$$
k_{j} \int_{\Omega} \sigma_{h, j}: \varepsilon\left(v_{h}\right) \mathrm{d} x=\int_{t_{j-1}}^{t_{j}} \int_{\Omega} f(t) \cdot v_{h} \mathrm{~d} x \mathrm{~d} t+\int_{t_{j-1}}^{t_{j}} \int_{\Gamma_{\mathrm{N}}} g(t) \cdot v_{h} \mathrm{~d} s \mathrm{~d} t
$$

for all $v_{h} \in \mathscr{S}_{\mathrm{D}}^{1}(\mathscr{T})$ and $\Phi\left(\sigma_{h, j}, \chi_{h, j}\right) \leqslant 0$ and, for all $\left(\tau_{h}, \psi_{h}\right) \in \mathbb{L}(\mathscr{T})$ with $\Phi\left(\tau_{h}, \psi_{h}\right) \leqslant 0$, there holds

$$
\int_{\Omega}\left(\varepsilon\left(u_{h, j}-u_{h, j-1}\right)-\mathbb{C}^{-1}\left(\sigma_{h, j}-\sigma_{h, j-1}\right)\right):\left(\tau_{h}-\sigma_{h, j}\right) \mathrm{d} x-\int_{\Omega}\left(\chi_{h, j}-\chi_{h, j-1}\right) \cdot \mathbb{Q}^{-1}\left(\psi_{h}-\chi_{h, j}\right) \mathrm{d} x \leqslant 0 .
$$

Theorem 5.1. The discrete problems $\mathrm{dG}(0)$ for the single-step primal and dual formulation have unique discrete solutions. The resulting piecewise constant approximation

$$
\left(u_{h}, P_{h}\right) \in \mathscr{L}^{0}\left(\mathscr{I} ; \mathscr{S}^{1}(\mathscr{T}) \times \mathbb{L}(\mathscr{T})\right)
$$

and $\left(u_{h}, \Sigma_{h}\right) \in \mathscr{L}^{0}\left(\mathscr{I} ; \mathscr{S}^{1}(\mathscr{T}) \times \llbracket(\mathscr{T})\right)$ solves the $\mathrm{dG}(0)$-discrete-primal and dual formulation, respectively.

Proof. Since the $\mathrm{dG}(0)$ single-step versions can be regarded as bE scheme with a modified right-hand side ( $f$ and $g$ ), there exist solutions for the same reasons that guarantee the feasibility of the bE scheme. Then, it is not hard to see that the resulting respective $\mathscr{I}$ piecewise constant vectors solve the (total-step) discrete formulations. (Recall for the primal problem that $\operatorname{supp}_{K}$ is homogeneous of degree 1 and so may be multiplied with the time-step $k_{j}$ followed by a change of test-functions.) 
More notation is required to present more details on $\mathrm{dG}(1)$. In the time step for $I_{j}=\left(t_{A}, t_{B}\right)$, we are given $u_{0}, P_{0}, \Sigma_{0}$ as the left-sided limits at $t_{A}$ and seek an affine function $\left(u_{h}, P_{h}, \Sigma_{h}\right)$ in time,

$$
(u, P, \Sigma)(t)=\left(u_{A}, P_{A}, \Sigma_{A}\right)+\left(t-t_{A}\right) / k\left(u_{B}-u_{A}, P_{B}-P_{A}, \Sigma_{B}-\Sigma_{A}\right) \text { for } t_{A}<t \leqslant t_{B} .
$$

Let $\left(\varphi_{1}, \ldots, \varphi_{n}\right)$ be a (standard) nodal basis of $\mathscr{S}_{\mathrm{D}}^{1}(\mathscr{T})^{d} \subseteq H_{\mathrm{D}}^{1}(\Omega)$ and define the discrete right-hand sides $F=\left(F_{j}^{1}, F_{j}^{2}\right) \in \mathbb{R}^{n \times 2}$ by

$$
\begin{aligned}
& F_{\ell}^{1}:=\int_{t_{j-1}}^{t_{j}} \int_{\Omega}\left(t-t_{A}\right) / k \varphi_{\ell} \cdot f(t) \mathrm{d} x \mathrm{~d} t+\int_{t_{j-1}}^{t_{j}} \int_{\Gamma_{\mathrm{N}}}\left(t-t_{A}\right) / k \varphi_{\ell} \cdot g(t) \mathrm{d} s \mathrm{~d} t \\
& F_{\ell}^{2}:=\int_{t_{j-1}}^{t_{j}} \int_{\Omega}\left(t_{B}-t\right) / k \varphi_{\ell} \cdot f(t) \mathrm{d} x \mathrm{~d} t+\int_{t_{j-1}}^{t_{j}} \int_{\Gamma_{\mathrm{N}}}\left(t_{B}-t\right) / k \varphi_{\ell} \cdot g(t) \mathrm{d} s \mathrm{~d} t
\end{aligned}
$$

for all $\ell=1, \ldots, n$. The scalar product in time is described by the fourth-order tensor $\mathbb{M}$ and the corresponding bilinear form

$$
\begin{aligned}
& (A, B) \mathbb{M}(C, D):=k / 6(A: B+2 B: C+2 A: D+B: D) \quad \text { for } A, B, C, D \in \mathbb{R}_{\mathrm{sym}}^{d \times d}, \\
& (A, B) \mathbb{M}(C, D):=k / 6(A \star B+2 B \star C+2 A \star D+B \star D) \quad \text { for } A, B, C, D \in \mathbb{R}_{\mathrm{sym}}^{d \times d} \times \mathbb{R}^{m} .
\end{aligned}
$$

Then, the time step in the $\mathrm{dG}(k)$-discrete dual formulation reads, in the above notation as follows.

One time step in $d G(1)$-dual formulation: Seek $u_{A}, u_{B} \in \mathscr{S}^{1}(\mathscr{T})^{d}$ with $u_{A}=u_{\mathrm{D}, h}\left(t_{A}\right), u_{B}=u_{\mathrm{D}, h}\left(t_{B}\right)$ on $\Gamma_{\mathrm{D}}$ and $\Sigma_{A}, \Sigma_{B} \in \mathbb{L}(\mathscr{T})$ with

$$
\Phi\left(\Sigma_{A}\right) \leqslant 0, \quad \Phi\left(\Sigma_{B}\right) \leqslant 0, \quad \Sigma_{A}=\left(\sigma_{A}, \chi_{A}\right), \quad \Sigma_{B}=\left(\sigma_{B}, \chi_{B}\right)
$$

such that

$$
\int_{\Omega}\left(\sigma_{A}, \sigma_{B}\right) \mathbb{M}\left(\varepsilon\left(\varphi_{j}\right), \varepsilon\left(\varphi_{k}\right)\right) \mathrm{d} x=\left(F_{j}^{1}+F_{k}^{2}\right) \quad(j, k=1, \ldots, n)
$$

and, for each $T \in \mathscr{T}$ and all $T_{A}=\left(\tau_{A}, \psi_{A}\right), T_{B}=\left(\tau_{B}, \psi_{B}\right) \in \mathbb{R}_{\mathrm{sym}}^{d \times d} \times \mathbb{R}^{m}$ with $\Phi\left(T_{A}\right) \leqslant 0, \Phi\left(T_{B}\right) \leqslant 0$ and for $P_{0}:=\left.\left(\varepsilon\left(u_{0}\right)-\mathbb{C}^{-1} \sigma_{0},-\mathbb{T}^{-1} \chi_{0}\right)\right|_{T}, P_{A}=\left.\left(\varepsilon\left(u_{A}\right)-\mathbb{C}^{-1} \sigma_{A},-\mathbb{T}^{-1} \chi_{A}\right)\right|_{T}, P_{B}:=\left.\left(\varepsilon\left(u_{B}\right)-\mathbb{C}^{-1} \sigma_{B},-\mathbb{H}^{-1} \chi_{B}\right)\right|_{T} \in \mathbb{R}_{\mathrm{sym}}^{d \times d} \times$ $\mathbb{R}^{m}$, there holds

$$
1 / 2\left(P_{B}-P_{A}\right) \star\left(T_{A}-\Sigma_{A}+T_{B}-\Sigma_{B}\right)+\left(P_{A}-P_{0}\right) \star\left(T_{A}-\Sigma_{A}\right) \leqslant 0 .
$$

The time integration of the supp term is slightly more involved and discussed below.

One time step in $d G(1)$ primal formulation: Seek $u_{A}, u_{B} \in \mathscr{S}^{1}(\mathscr{T})^{d}$ with $u_{A}=u_{\mathrm{D}, h}\left(t_{A}\right), u_{B}=u_{\mathrm{D}, h}\left(t_{B}\right)$ on $\Gamma_{\mathrm{D}}$ and $P_{A}=\left(p_{A}, \xi_{A}\right), P_{B}=\left(p_{B}, \xi_{B}\right) \in \mathbb{L}(\mathscr{T})$ such that

$$
\begin{array}{ll}
\sigma_{A}:=\left(\mathbb{C} \varepsilon\left(u_{A}\right)-p_{A}\right), & \sigma_{B}:=\left(\mathbb{C} \varepsilon\left(u_{B}\right)-p_{B}\right), \\
\Sigma_{A}:=\left.\left(\sigma_{A},-\mathbb{H} \xi_{A}\right)\right|_{T}, & \Sigma_{B}:=\left.\left(\sigma_{B},-\mathbb{M} \xi_{B}\right)\right|_{T} \in \mathbb{R}_{\mathrm{sym}}^{d \times d} \times \mathbb{R}^{m}
\end{array}
$$

satisfy

$$
\int_{\Omega}\left(\sigma_{A}, \sigma_{B}\right) \mathbb{M}\left(\varepsilon\left(\varphi_{j}\right), \varepsilon\left(\varphi_{k}\right)\right) \mathrm{d} x=\left(F_{j}^{1}+F_{k}^{2}\right) \quad(j, k=1, \ldots, n)
$$

and, for each $T \in \mathscr{T}$ and all $Q_{A}, Q_{B} \in \mathbb{R}_{\mathrm{sym}}^{d \times d} \times \mathbb{R}^{m}$,

$$
\begin{aligned}
& \left(\Sigma_{A}, \Sigma_{B}\right) \mathbb{M}\left(Q_{A}, Q_{B}\right)-1 / 2\left(\Sigma_{A}+\Sigma_{B}\right) \star\left(P_{B}-P_{A}\right)-\Sigma_{A} \star\left(P_{A}-P_{0}\right) \\
& \quad \leqslant \sup _{\substack{\Phi\left(R_{A}\right) \leqslant 0 \\
\Phi\left(R_{B}\right) \leqslant 0}}\left(Q_{A}, Q_{B}\right) \mathbb{M}\left(R_{A}, R_{B}\right)-\sup _{\substack{\Phi\left(R_{A}\right) \leqslant 0 \\
\Phi\left(R_{B}\right) \leqslant 0}}\left(1 / 2\left(P_{B}-P_{A}\right) \star\left(R_{A}+R_{B}\right)+\left(P_{A}-P_{0}\right) \star R_{A}\right) .
\end{aligned}
$$


Example 5.1 (linear kinematic hardening). The von-Mises yield function

$$
\Phi: \mathbb{R}_{\mathrm{sym}}^{d \times d} \times \mathbb{R}^{m} \rightarrow \mathbb{R}
$$

is written for $\mathbb{R}^{m} \equiv \mathbb{R}_{\text {sym }}^{d \times d}$ (i.e., the $m=d(d+1) / 2$ entries of $\mathbb{R}^{m}$ are ordered canonically to define a symmetric $d \times d$ matrix),

$$
\Phi(\sigma, \chi)=|\operatorname{dev}(\sigma+\chi)|-\sqrt{2 / 3} \sigma_{y} .
$$

Then

$$
\operatorname{supp}_{K}(p, \xi)= \begin{cases}\sqrt{2 / 3} \sigma_{y}|p| & \text { if } \operatorname{tr}(p)=0 \text { and } p=\xi \\ +\infty & \text { else. }\end{cases}
$$

Furthermore, for any $S_{A}=\left(p_{A}, \xi_{A}\right), S_{B}=\left(p_{B}, \xi_{B}\right) \in \mathbb{R}_{\mathrm{sym}}^{d \times d} \times \mathbb{R}^{m}$,

$$
\sup _{\substack{\Phi\left(R_{A}\right) \leqslant 0 \\ \Phi\left(R_{B}\right) \leqslant 0}}\left(R_{A} \star S_{A}+R_{B} \star S_{B}\right)= \begin{cases}\sqrt{2 / 3}\left(\left|p_{A}\right|+\left|p_{B}\right|\right) & \text { if } \operatorname{tr}\left(p_{A}\right)=\operatorname{tr}\left(p_{B}\right)=0, \\ & \text { and } p_{A}=\xi_{A}, p_{B}=\xi_{B}, \\ & \text { else. }\end{cases}
$$

This enables a direct evaluation of the inequality in one time step in the primal formulation.

In order to simplify the variational inequality further, observe that $\mathbb{M}\left(Q_{A}, Q_{B}\right)$ is an arbitrary test functional (i.e., the linear operator $\mathscr{M}$ behind the bilinear form associated with $\mathbb{M}$ is bijective). Hence $\mathbb{M}\left(Q_{A}, Q_{B}\right)$ may be substituted by $\left(Q_{A}, Q_{B}\right)$. This and direct calculations show

$$
\begin{aligned}
& \Sigma_{A} \star\left(Q_{A}-\left(P_{B}+P_{A}\right) / 2+P_{0}\right)+\Sigma_{B} \star\left(Q_{B}-\left(P_{B}-P_{A}\right) / 2\right) \\
& \quad \leqslant \operatorname{supp}_{K}\left(Q_{A}\right)-\operatorname{supp}_{K}\left(\left(P_{B}+P_{A}\right) / 2-P_{0}\right)+\operatorname{supp}_{K}\left(Q_{B}\right)-\operatorname{supp}_{K}\left(\left(P_{B}-P_{A}\right) / 2\right)
\end{aligned}
$$

for all $Q_{A}, Q_{B} \in \mathbb{R}_{\mathrm{sym}}^{d \times d} \times \mathbb{R}^{m}$. This system is equivalent to two separate variational inequalities which, in terms of subgradients in convex analysis, read

$$
\Sigma_{A} \in \partial \operatorname{supp}_{K}\left(\left(P_{A}+P_{B}\right) / 2-P_{0}\right) \quad \text { and } \quad \Sigma_{B} \in \partial \operatorname{supp}_{K}\left(\left(P_{B}-P_{A}\right) / 2\right) .
$$

Example 5.2 (linear kinematic hardening). In continuation of Example 5.1, we assume that $\because$ is $H$ times the identity and remark that the inclusions for

$$
\Sigma_{A}=\left(\mathbb{C}\left(\varepsilon\left(u_{A}\right)-p_{A}\right),-H \xi_{A}\right) \quad \text { and } \quad \Sigma_{B}=\left(\mathbb{C}\left(\varepsilon\left(u_{B}\right)-p_{B}\right),-H \xi_{B}\right) .
$$

lead to explicit representations. Indeed, the conditions on $p_{A}, p_{B}, \xi_{A}, \xi_{B} \in \mathbb{R}_{\mathrm{sym}}^{d \times d}$ read

$$
\begin{aligned}
& p_{A}=\xi_{A}, \quad p_{B}=\xi_{B}, \quad \operatorname{tr}\left(p_{A}\right)=\operatorname{tr}\left(p_{B}\right)=0, \\
& \operatorname{dev} \mathbb{C} \varepsilon\left(u_{A}\right)-(2 \mu+H) p_{A} \in \sqrt{2 / 3} \sigma_{y} \partial|\cdot|\left(\left(p_{B}+p_{A}\right) / 2-p_{0}\right), \\
& \operatorname{dev} \mathbb{C} \varepsilon\left(u_{B}\right)-(2 \mu+H) p_{B} \in \sqrt{2 / 3} \sigma_{y} \partial|\cdot|\left(\left(p_{B}-p_{A}\right) / 2\right) .
\end{aligned}
$$

The subgradient $\partial|\cdot|$ of the modulus function in $\mathbb{R}_{\mathrm{sym}}^{d \times d}$ reads $\partial|\cdot|(0)=\left\{q \in \mathbb{R}_{\mathrm{sym}}^{d \times d}:|q| \leqslant 1\right\}$ and $\partial|\cdot|(q)=$ $\{q /|q|\}$ for $q \in \mathbb{R}_{\mathrm{sym}}^{d \times d} \backslash\{0\}$, i.e.,

$$
q \in \partial|\cdot|(p) \Longleftrightarrow(p=0 \text { and }|q| \leqslant 1) \text { or }(p \neq 0 \text { and } q=p /|p|) \text {. }
$$

The example illustrates, that, given $u_{A}$ and $u_{B}$, the variational inequalities can be solved elementwise very directly for particular hardening laws. In the example we failed to give explicit formulae for $p_{A}$ and $p_{B}$ as a function of $\varepsilon\left(u_{A}\right), \varepsilon\left(u_{B}\right)$, and $p_{0}$. There is, however, the following algorithm to compute them. 
Algorithm 5.1 (computing $\left(p_{B}, p_{A}\right)$ in terms of $\left.\varepsilon\left(u_{A}\right), \varepsilon\left(u_{B}\right), p_{0}\right)$. In the notation of the example we are given material parameters $\mathbb{C}, H>0, \sigma_{y}>0$ and $\varepsilon\left(u_{A}\right), \varepsilon\left(u_{B}\right), p_{0}$.

(a) Set $\gamma:=\sqrt{2 / 3} \sigma_{y}, A_{1}:=\operatorname{dev} \mathbb{C} \varepsilon\left(u_{B}\right)-(2 \mu+H) p_{0}, A_{2}:=\operatorname{dev} \mathbb{C} \varepsilon\left(u_{A}\right)-(2 \mu+H) p_{0}$, and set $a:=\left|A_{1}\right| / \gamma$.

(b) If $\left|A_{1}\right|=\left|A_{2}\right|=0$ then set $\mu_{1}=0=\mu_{2}$ and goto (k).

(c) If $\left|A_{1}\right|=0<\left|A_{2}\right|=: \beta$ then set

$$
\mu_{1}=(\beta-2 \gamma)_{+} / \beta \text { and } \mu_{2}=\left(\beta^{2} / \gamma-\beta\right) /\left((\beta-2 \gamma)_{+}+\beta^{2} / \gamma\right)
$$

and goto $(\mathrm{k})$.

(d) If $0<\left|A_{1}\right|$ then set $b:=A_{1}: A_{2} /\left(\gamma\left|A_{1}\right|\right)$ and $c:=\left|A_{2}-b / a A_{1}\right| / \gamma$.

(e) If $c=0$ then follow exactly one of the following cases $\left(\mathrm{e}_{1}\right)-\left(\mathrm{e}_{4}\right)$

$\left(\mathrm{e}_{1}\right)$ If $(a-1)_{+}<\left|b-1+(1-a)_{+}\right|-2$ set $\mu_{1}=(|b-a|-2) /|b-a|$ and $\mu_{2}=(|b-a|+2 a) /$ $(|b-a|+2 a+2)$

(e $\left.\mathrm{e}_{2}\right)$ If $\left|b-1+(1-a)_{+}\right|-2 \leqslant(a-1)_{+}<|b|-1$ set $\mu_{1}=0, \mu_{2}=(|b|-1) /|b|$;

(e $\left.\mathrm{e}_{3}\right)$ If $|b|-1 \leqslant(a-1)_{+}<|b-1|$ set $\mu_{1}=(a-1)_{+} / a, \mu_{2}=0$;

$\left(\mathrm{e}_{4}\right)$ If $|b-1| \leqslant(a-1)_{+}$set $\mu_{1}=(a-b) /(a-b+2), \mu_{2}=(a+b-2) /(a+b)$, and goto $(\mathrm{k})$.

(f) If $c \neq 0$ set $\mu_{1}:=(a-1)_{+} / a$.

(g) If $\left(\mu_{1} a+b\right)^{2}+c^{2} \leqslant 1$ set $\mu_{2}:=0$ and goto (k).

(h) If $\left(\mu_{1} a+b\right)^{2}+c^{2}>1$ set $\mu_{2}:=\left(\sqrt{b^{2}+c^{2}}-1\right)_{+} / \sqrt{b^{2}+c^{2}}$.

(i) If $\left(a-\mu_{2} b\right)^{2}+\left(\mu_{2} c\right)^{2} \leqslant 1$ set $\mu_{1}:=0$ and goto $(\mathrm{k})$.

(j) If $\left(a-\mu_{2} b\right)^{2}+\left(\mu_{2} c\right)^{2}>1$ solve

$$
\begin{gathered}
{\left[(a(x+f(x))-b g(x))^{2}+c^{2} g(x)^{2}\right]^{1 / 2} x=\max \left\{0,\left[(a(x+f(x))-b g(x))^{2}+c^{2} g(x)^{2}\right]^{1 / 2}\right.} \\
-(x+f(x)+x g(x))\}
\end{gathered}
$$

for $x$ in $(0,1)$. Here, $f(x):=\sqrt{(a x+b)^{2}+c^{2}}$ and $g(x):=\max \{0, f(x)-1\}$. Set $\mu_{1}:=x$ for the solution $x$ and $\mu_{2}:=g\left(\mu_{1}\right) /\left(\mu_{1}+f\left(\mu_{1}\right)\right)$.

(k) Given $\mu_{1}, \mu_{2}$ in $[0,1)$, let

$$
\begin{aligned}
& p_{A}:=\left(\left(A_{1}+A_{2}\right) \mu_{1} \mu_{2}-\mu_{1} A_{1}+\mu_{2} A_{2}\right) /\left(\left(1+\mu_{1} \mu_{2}\right)(2 \mu+H)\right)+p_{0}, \\
& p_{B}:=\left(\left(A_{1}-A_{2}\right) \mu_{1} \mu_{2}+\mu_{1} A_{1}+\mu_{2} A_{2}\right) /\left(\left(1+\mu_{1} \mu_{2}\right)(2 \mu+H)\right)+p_{0} .
\end{aligned}
$$

Theorem 5.2. Given $\varepsilon\left(u_{A}\right), \varepsilon\left(u_{B}\right), p_{0}$ in Example 5.2, the Algorithm 5.1 computes $p_{A}$ and $p_{B}$. Then, $\Sigma_{A}$ and $\Sigma_{B}$ which are the unique solutions of

$$
\Sigma_{A} \in \partial \operatorname{supp}_{K}\left(\left(P_{A}+P_{B}\right) / 2-P_{0}\right) \quad \text { and } \quad \Sigma_{B} \in \partial \operatorname{supp}_{K}\left(\left(P_{B}-P_{A}\right) / 2\right) .
$$

Proof. The proof consists of laborious but essentially direct analytical considerations on the solution $\mu_{1}$ and $\mu_{2}$ of

$$
\begin{aligned}
& \mu_{1}\left|A_{1}-\mu_{2} A_{2}\right|=\left(\left|A_{1}-\mu_{2} A_{2}\right|-\gamma\left(1+\mu_{1} \mu_{2}\right)\right)_{+}, \\
& \mu_{2}\left|\mu_{1} A_{1}+A_{2}\right|=\left(\left|\mu_{1} A_{1}+A_{2}\right|-\gamma\left(1+\mu_{1} \mu_{2}\right)\right)_{+} .
\end{aligned}
$$

We mention that $0 \leqslant \mu_{1}, \mu_{2}<1$ is always guaranteed and refer to [1] for further details.

The two time-step formulations for $\mathrm{dG}(1)$ have unique solutions. The computation of which employs an alternating algorithm. 
Algorithm 5.2 (computing the discrete solution). In the notation of Example 5.1 and of the dG(1)-Primal Formulation, initialize $\left(p_{A}, p_{B}\right):=\left(p_{0}, p_{0}\right)$ on each $T \in \mathscr{T}$.

(a) Given $\left(p_{A}, p_{B}\right)$ solve the linear system of equations

$$
\int_{\Omega}\left(\mathbb{C} \varepsilon\left(u_{A}\right), \mathbb{C} \varepsilon\left(u_{B}\right)\right) \mathbb{M}\left(\varepsilon\left(\varphi_{j}\right), \varepsilon\left(\varphi_{k}\right)\right) \mathrm{d} x=\left(F_{j}^{1}+F_{k}^{2}\right)+\int_{\Omega}\left(p_{A}, p_{B}\right) \mathbb{M}\left(\varepsilon\left(\varphi_{j}\right), \varepsilon\left(\varphi_{k}\right)\right) \mathrm{d} x
$$

for $j, k=1, \ldots, n$ and $\left(u_{A}, u_{B}\right) \in\left(u_{\mathrm{D}, h}\left(t_{A}\right), u_{\mathrm{D}, h}\left(t_{B}\right)\right)+\mathscr{S}_{\mathrm{D}}^{1}(\mathscr{T})^{2}$.

(b) Given $\left(u_{A}, u_{B}\right)$ and $T \in \mathscr{T}$ compute $\left(\left.p_{A}\right|_{T},\left.p_{B}\right|_{T}\right)$ from $\left.\varepsilon\left(u_{A}\right)\right|_{T},\left.\varepsilon\left(u_{B}\right)\right|_{T},\left.p_{0}\right|_{T}$ with Algorithm 5.1.

(c) Check convergence and terminate with (d) or continue with (a).

(d) Output is $u_{A}, u_{B}, P_{A}, P_{B}$ and $\Sigma_{A}, \Sigma_{B}$.

Remark 5.1 (computing the discrete solution for dual formulation). An Uzawa-type algorithm is suggested for the $\mathrm{dG}(1)$ single-step dual formulation where Step (b) of Algorithm 5.2 is replaced by a regularized version of the equilibrium equations, namely, for $j=1, \ldots, n$,

$$
\int_{\Omega}\left(\varepsilon\left(u_{A}\right), \varepsilon\left(u_{B}\right)\right) \mathbb{M}\left(\varepsilon\left(\varphi_{j}\right), \varepsilon\left(\varphi_{k}\right)\right) \mathrm{d} x=\left(F_{j}^{1}+F_{k}^{2}\right)-\int_{\Omega}\left(\sigma_{A}, \sigma_{B}\right) \mathbb{M}\left(\varepsilon\left(\varphi_{j}\right), \varepsilon\left(\varphi_{k}\right)\right) \mathrm{d} x
$$

is solved for $\left(u_{A}, u_{B}\right)$ and $\left(\sigma_{A}, \sigma_{B}\right)$ is then computed elementwise by direct solutions of the variational inequalities in the particular situation.

Remark 5.2 (convergence of Algorithm 5.2). A convergence proof of Algorithm 5.2 might be possible along the arguments from [6]. In the numerical examples of Section 6 we obtained global convergence with up to a few hundred iterations even for the finest meshes to achieve a residual vector smaller than $10^{-3}$. More efficient multilevel or domain decomposition techniques are desirable in the future.

Remark $5.3(\mathrm{dG}(k)$ for $k \geqslant 2)$. The main obstacle for $\mathrm{dG}(k)$ for $k \geqslant 2$ consists in the side restriction $\Phi(\Sigma(t)) \leqslant 0$ for all $t \in I_{j}$. If $k \leqslant 1$, this is a simple consequence of $\Phi\left(\Sigma_{A}\right) \leqslant 0, \Phi\left(\Sigma_{B}\right) \leqslant 0$, and the convexity of $\Phi$. For $k \geqslant 2$ this is no longer as simple as this.

\section{Numerical examples}

This section is devoted to two applications, Cook's membrane and a perforated tension strip. The elastoplastic material is adopted from Example 5.1 and Algorithm 5.2 is used for the computations.

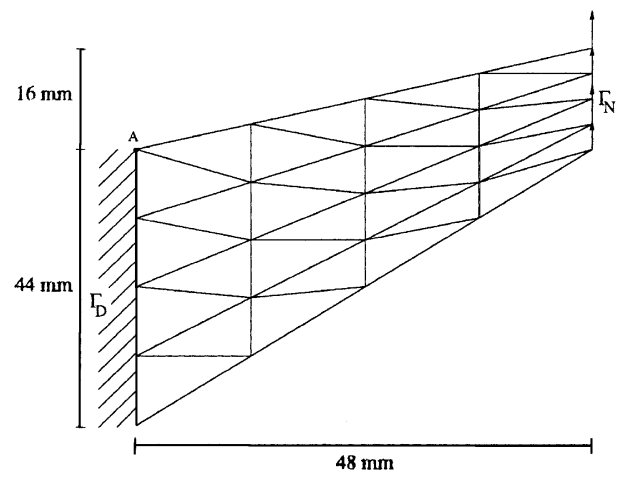

$$
\begin{aligned}
E & =100.000 \quad \nu=\frac{1}{3} \\
\mu & =\frac{E}{2(1+\nu)} \quad \lambda=\frac{\nu E}{(1+\nu)(1-2 \nu)} \\
\sigma_{y} & =2,2 \quad \mathbb{H}_{2}=1 \\
g(48, y, t) & = \begin{cases}(0,0) & \text { for } t<0 \\
(0, t) & \text { for } t \in[0,1] \\
(0,1) & \text { for } t>1\end{cases}
\end{aligned}
$$

Fig. 2. System and initial mesh $\mathscr{T}_{0}$ for Cook's membrane. 


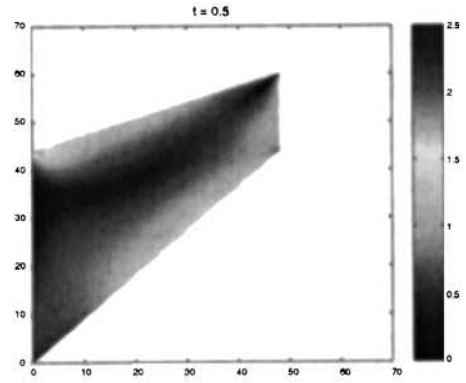

(a)

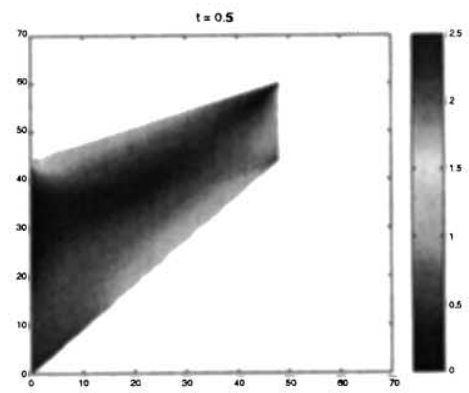

(c)

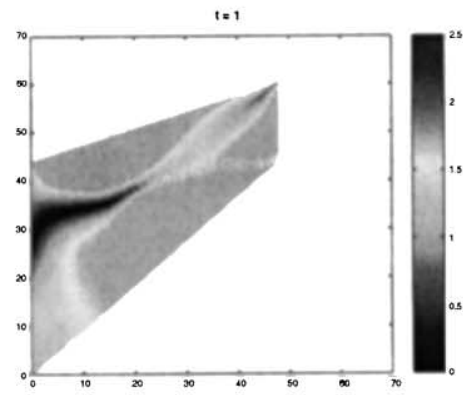

(e)

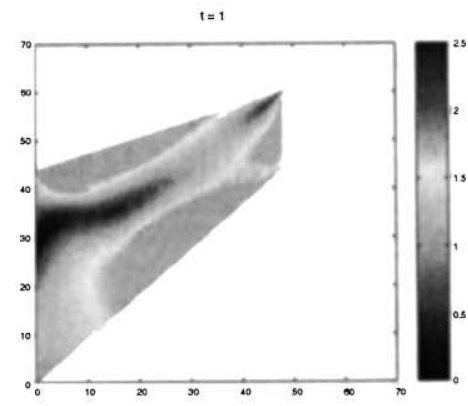

(g)

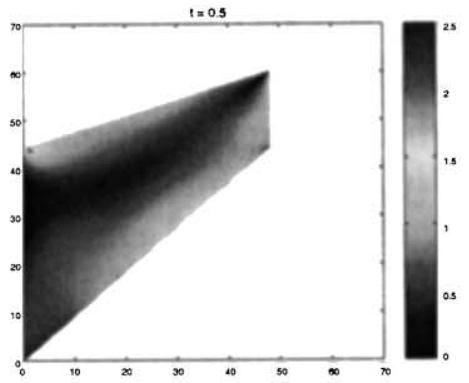

(b)

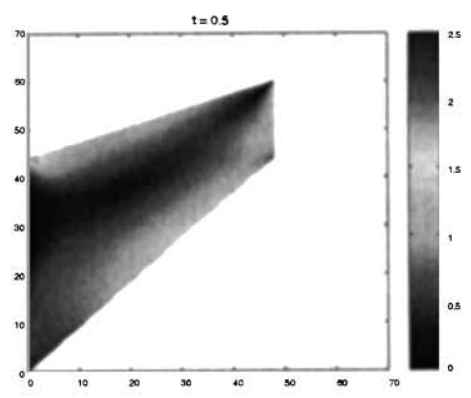

(d)

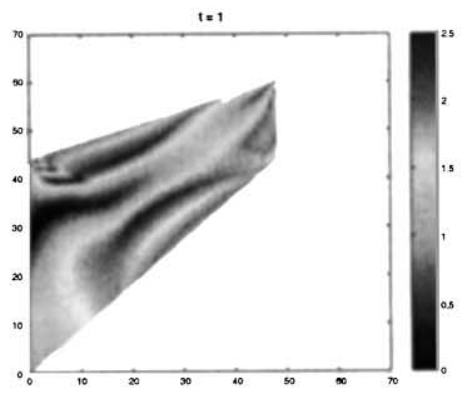

(f)

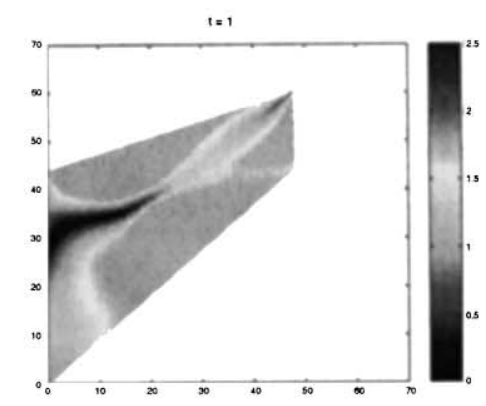

(h)

Fig. 3. Calculated deformed configuration for $t=1 / 2$ and $t=1$ in Example 6.1 with von-Mises stresses in color: (a) bE, $t=1 / 2$; (b) $\mathrm{CN}, t=1 / 2$; (c) $\mathrm{dG}(0), t=1 / 2$; (d) $\mathrm{dG}(1), t=1 / 2$; (e) bE, $t=1$; (f) $\mathrm{CN}, t=1$; (g) $\mathrm{dG}(0), t=1$; (h) $\mathrm{dG}(1), t=1$. 


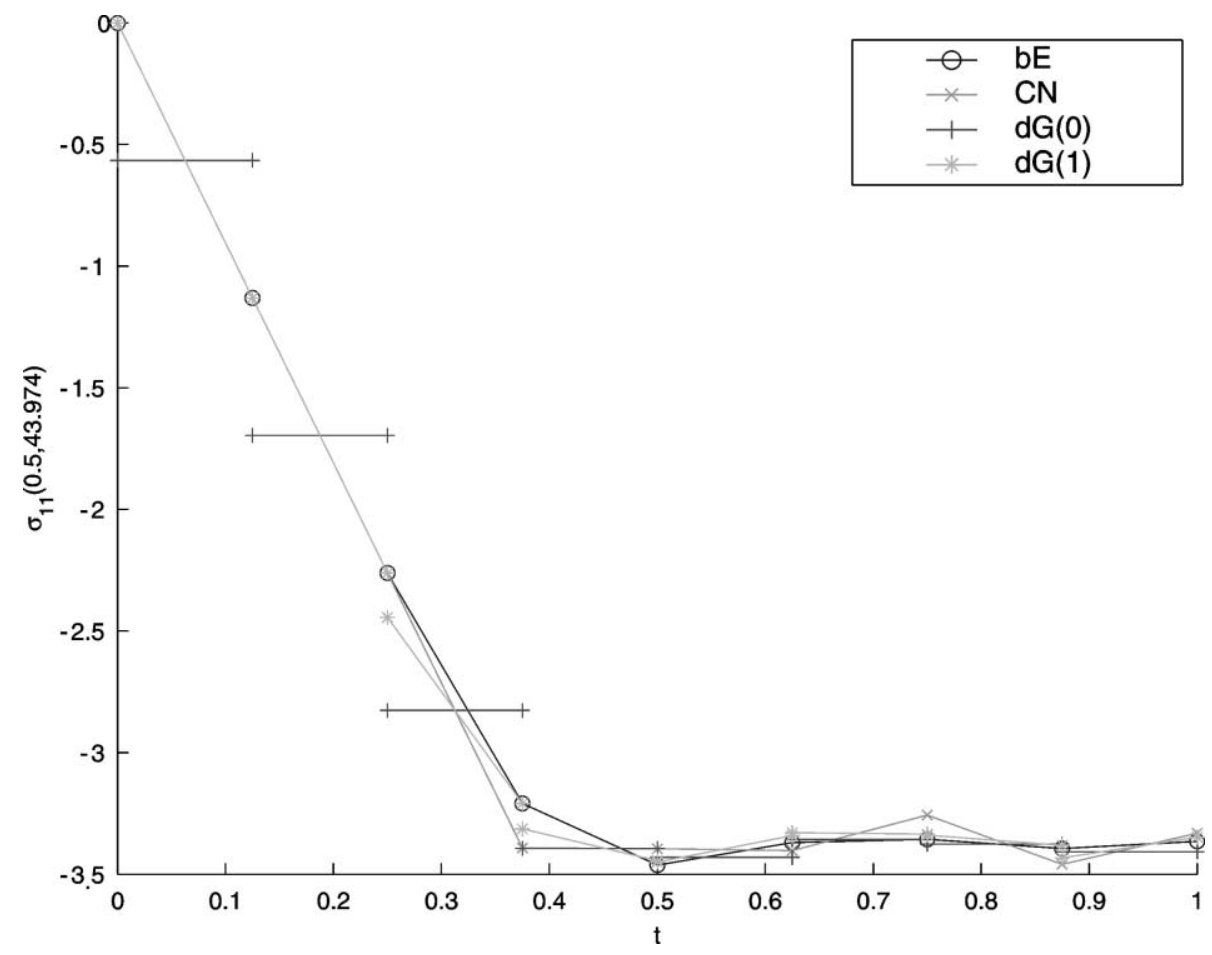

Fig. 4. History of discrete stress component $\sigma_{11}(0.5000,43.9740, t)$ at a point $\mathrm{A}^{\prime}$ as a function of time in the time interval $[0,1]$.

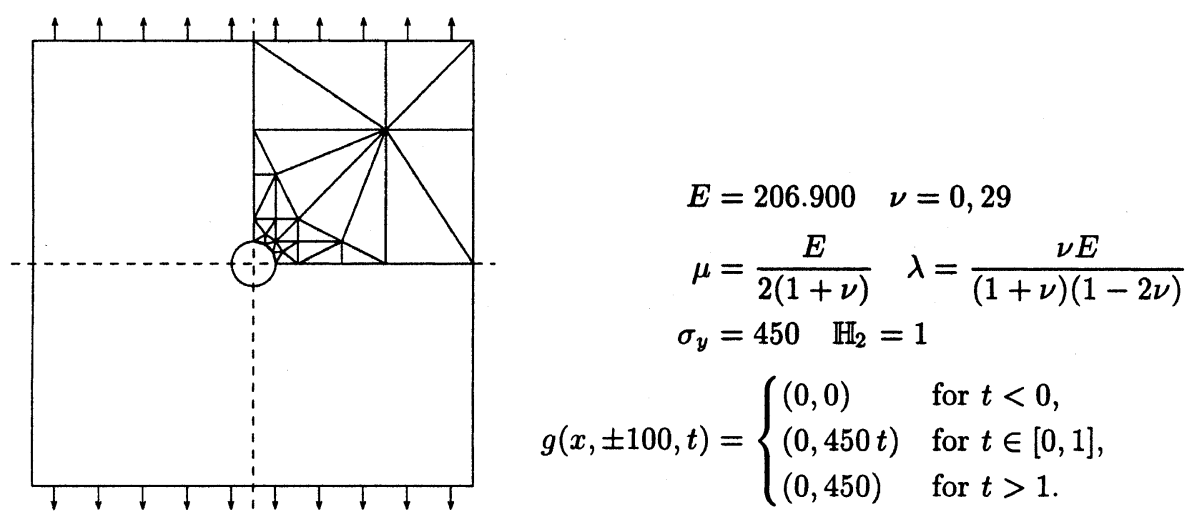

Fig. 5. System and initial mesh $\mathscr{T}_{0}$ in Example 6.2.

\subsection{Cook membrane}

The 2D elastoplastic quadrilateral body $\Omega=\operatorname{conv}\{A, B, C, D\}$ is defined for $A=(0,44), B=(0,0)$, $C=(48,44), D=(48,60)$. The system, its Dirichlet boundary $\Gamma_{\mathrm{D}}=\operatorname{conv}\{A, B\}$, and its loading $g(t)$ in vertical direction along $\operatorname{conv}\{C, D\}$ while $g \equiv 0$ along $\operatorname{conv}\{B, C\} \cup \operatorname{conv}\{D, A\}$, is depicted in Fig. 2 together with the initial triangulation $\mathscr{T}_{0}$ and the material parameter. There is no volume force $(f \equiv 0$ in $\Omega$ ) 


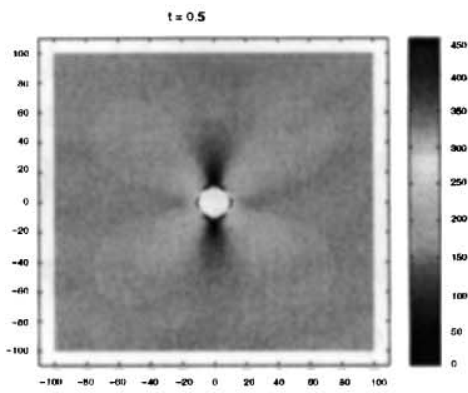

(a)

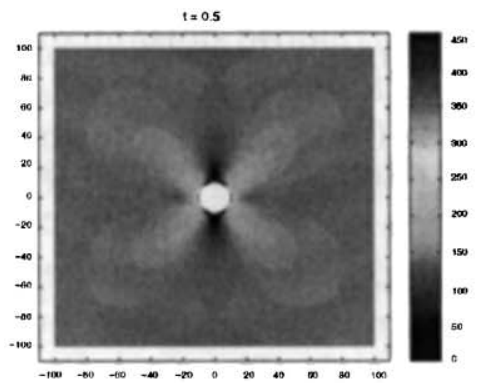

(c)

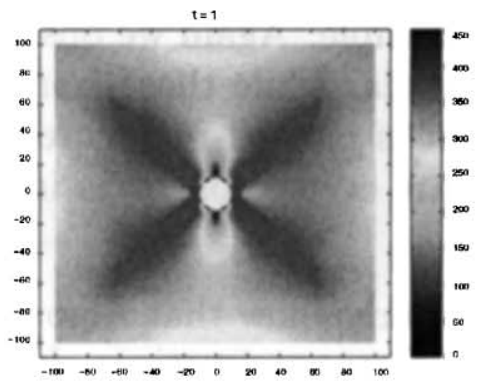

(e)

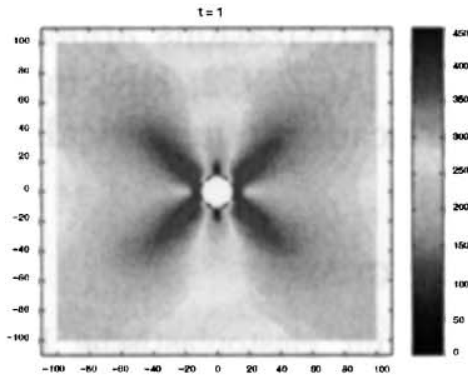

(g)

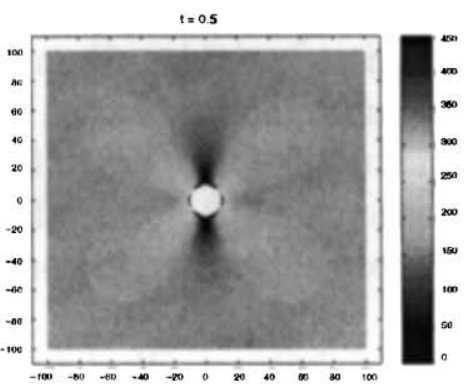

(b)

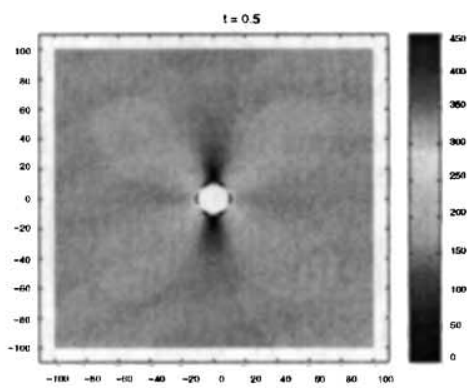

(d)

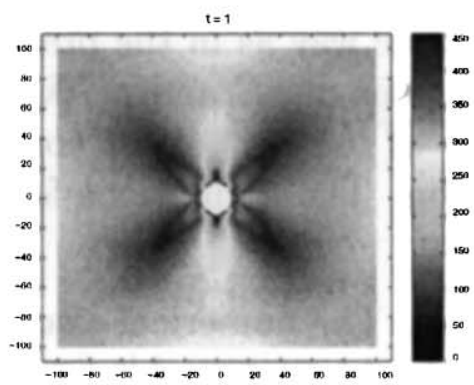

(f)

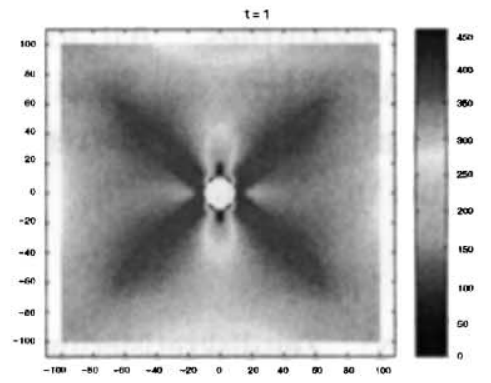

(h)

Fig. 6. Calculated deformed configuration for $t=1 / 2$ and $t=1$ in Example 6.2 with von-Mises stresses in color: (a) bE, $t=1 / 2$; (b) $\mathrm{CN}, t=1 / 2$; (c) $\mathrm{dG}(0), t=1 / 2$; (d) $\mathrm{dG}(1), t=1 / 2$; (e) bE, $t=1$; (f) $\mathrm{CN}, t=1$; (g) $\mathrm{dG}(0), t=1$; (h) $\mathrm{dG}(1), t=1$. 
and the applied surface load $g(t)$ is considered for a process time $t$ in the time domain $(0,1)$ with homogeneous initial and boundary conditions $\left(u_{\mathrm{D}}=0\right)$.

The time discretization employed eight uniform time steps with constant time increment 0.125 with a mesh $\mathscr{T}_{3}$ obtained by successive red-refinements starting with $\mathscr{T}_{0}$ from Fig. 2. (A red-refinement of a triangle $T$ is the division of $T$ into four congruent sub-triangles derived from lines through the edges' midpoints.) Fig. 3 displays the eight numerical results obtained with $\mathrm{bE}, \mathrm{CN}, \mathrm{dG}(0), \mathrm{dG}(1)$ for $t=1 / 2$ and $t=1$. The displacements are magnified by a factor 20 to show the deformed configuration. The grey tones inside display the von-Mises stress

$$
\sigma_{\mathrm{ref}}:=\left|\operatorname{dev}\left(\sigma_{h}+\chi_{h}\right)\right|
$$

The results for $t=1 / 2$ are comparable while, for $t=1$, we observe differences. The von-Mises stresses of $\mathrm{bE}$ and $\mathrm{dG}(1)$ are comparable and similar to that of $\mathrm{dG}(0)$. The values for $\mathrm{CN}$ appear different. To explore the reasons for this behavior and to illustrate the respective time approximation properties of the four schemes, Fig. 4 displays the discrete stress component $\left(\sigma_{h}\right)_{11}$ at the point $A^{\prime}=(0.5,43.974)$, center of inertia of a finite element close to $A$, as a function of time $t$ for $0 \leqslant t \leqslant 1$. The material behavior near $A$ seems to be elastic for $0 \leqslant t \leqslant 0.4$. Since the plastic behavior starts near $A$, we deduce that the overall material behavior is rather elastic for $t=1 / 2$ which explains the similar von-Mises stress fields computed by all four schemes for $t=1 / 2$. In contrast, for $t=1$, we have large parts of the body in the plastic state and the values for $\sigma_{11}$ near $A$ are almost constant. While $\mathrm{bE}, \mathrm{dG}(0), \mathrm{dG}(1)$ are almost constant, the $\mathrm{CN}$ approximations oscillate around the constant value. As mean value is met at the mid-point of the time interval $I_{8}$, we have a perturbation for $t=T=1$. Our interpretation is that Fig. 4 shows oscillations for $t=1$ in the CN approximation which vanish in time and vanish in time averages.

\subsection{Perforated tension strip}

The second example corresponds to a benchmark [11] shown in Fig. 5. A squared domain $(100,+100)^{2}$ without a centered circular hole of radius 10 , i.e., $\Omega=(-100,+100)^{2} \backslash B(0,10)$, is pulled at the upper and lower ends by an applied surface load $g ; f \equiv 0$. Because of symmetry, only one quarter of the domain is discretized with an initial mesh $\mathscr{T}_{0}$ shown in Fig. 5. The time discretization in 8 uniform time steps with $k=1 / 8$ is as in the previous example, while the finite element mesh $\mathscr{T}_{4}$ with $N=8048$ degrees of freedom is generated by four successive red-refinements of $\mathscr{T}_{0}$. The eight approximations for $t=1 / 2$ and $t=1$ computed by $\mathrm{bE}, \mathrm{CN}, \mathrm{dG}(0), \mathrm{dG}(1)$ are shown in Fig. 6 in the same way as in the previous example. Again we observe that the von-Mises stress approximations of $\mathrm{bE}$ and $\mathrm{dG}(k)$ are similar while those from CN appear different. The time history of the (11) stress component is plotted in Fig. 1 which was discussed in the introduction. The oscillations for $\mathrm{CN}$ are even stronger as in the Cook's membrane problem.

\subsection{Conclusions}

The numerical examples clearly show that Algorithm 5.2 is feasible and $\mathrm{dG}(0)$ and $\mathrm{dG}(1)$ lead to reasonable finite element approximations. In contrast to the $\mathrm{CN}$ scheme, the higher order $\mathrm{dG}(1)$ does not show oscillations. This is important if one wants a hierarchy of schemes for the use of multilevel refinement indicators. The examples might suggest that $\mathrm{dG}(1)$ is the best amongst all four $\mathrm{bE}, \mathrm{CN}, \mathrm{dG}(0)$, and $\mathrm{dG}(1)$ schemes in the two examples. All four time discretizations appear to converge in time. This is proven in $[2,12,14]$ in time and in $[4,7,12]$ in space for $\mathrm{bE}$ and $\mathrm{CN}$. A corresponding analysis with a corresponding academic numerical example for $\mathrm{dG}(0)$ and $\mathrm{dG}(1)$ will be found in [3]. 


\section{References}

[1] J. Alberty, Zeitdiskretisierungsverfahren für elastoplastische Probleme der Kontinuumsmechanik Ph.D. thesis, University of Kiel, FRG, Tenea Verlag, 2001.

[2] J. Alberty, C. Carstensen, Numerical analysis of time-depending primal elastoplastic with hardening, SIAM J. Numer. Anal. 37 (4) (2000) 1271-1294.

[3] C. Carstensen, J. Alberty, Discontinuous Galerkin Time Discretization for Elastoplasticity: Convergence Analysis, in preparation.

[4] J. Alberty, C. Carstensen, D. Zarrabi, Adaptive numerical analysis in primal elastoplasticity with hardening, Comput. Methods Appl. Mech. Engrg. 171 (1999) 175-204.

[5] S.C. Brenner, L.R. Scott, The Mathematical Theory of Finite Element Methods, Springer Verlag, Berlin, 1994.

[6] C. Carstensen, Domain decomposition for a non-smooth convex minimisation problem and its application to plasticity, NLAA 4 (3) (1997) 1-13.

[7] C. Carstensen, Numerical analysis of primal elastoplasticity with hardening, Numer. Math. 82 (1999) $577-597$.

[8] P.G. Ciarlet, The Finite Element Method for Elliptic Problems, North-Holland, Amsterdam, 1978.

[9] L.C. Evans, Partial differential equations, in: Graduate Studies in Mathematics, 19, American Mathematical Society, Providence, RI, 1998.

[10] I. Ekeland, R. Temam, Convex Analysis and Variational Problems, North-Holland, Amsterdam, 1976.

[11] W. Hackbusch, E. Ramm, E. Rank, R. Rannacher, E. Stein, P. Wriggers, W.L. Wendland, K. Schweizerhof, Benchmark perforated tension strip. Communication in talk at ENUMATH Conference in Heidelberg, 1997. Available from <http:// www.ibnm.uni-hannover.de/forschung/paketantrag/benchmarks/benchmark.html>.

[12] W. Han, B.D. Reddy, Computational plasticity: the variational basis and numerical analysis, Comput. Mech. Adv. 2 (1995) 285400.

[13] C. Johnson, Existence theorems for plasticity problems, J. Math. Pures Appl. 55 (1976) 431-444.

[14] C. Johnson, A mixed finite element method for plasticity problems with hardening, SIAM J. Numer. Anal. 14 (1977) $575-583$.

[15] C. Johnson, On plasticity with hardening, J. Math. Anal. Appl. 62 (1978) 325-336.

[16] C. Johnson, P. Hansbo, Adaptive finite element methods in computational mechanics, Comput. Methods Appl. Mech. Engrg. 101 (1992) 143-181.

[17] G.A. Seregin, Differentiability of extremals of variational problems in the mechanics of perfect viscoplastic media, Diff. Equat. 23 (1987) 1349-1358.

[18] G.A. Seregin, On the regularity of the minimizers of some variational problems of plasticity theory, St. Petersburg Math. J. 4 (1993) 989-1020.

[19] E.M. Stein, Al. Idesman, R. Niekamp, Continuous and discontinuous Galerkin methods with finite elements in space and time for parallel computing of viscolelastic deformation, Comput. Methods Appl. Mech. Engrg. 190 (2000) 1049-1063.

[20] J.C. Simo, T.J.R. Hughes, Computational Inelasticity, Springer-Verlag, New York, 1998.

[21] T. Werder, K. Gerdes, D. Schötzau, C. Schwab, hp-Discontinuous Galerkin time stepping for parabolic problems, Comput. Methods Appl. Mech. Engrg. 190 (49-50) (2001) 6685-6708.

[22] E. Zeidler, Nonlinear Functional Analysis and its Applications III and IV, Springer-Verlag, New York, $1985,1988$. 